University of Nebraska - Lincoln

DigitalCommons@University of Nebraska - Lincoln

\title{
Impact of Porogens on the Pore Characteristics of Zirconia Particles Made by Polymer-Induced Colloid Aggregation
}

\author{
Abhinandan Pattanayak \\ University of Nebraska - Lincoln, abhi.pattanayak@gmail.com \\ Anuradha Subramanian \\ University of Nebraska - Lincoln, asubramanian2@unl.edu
}

Follow this and additional works at: https://digitalcommons.unl.edu/cbmesubramanian

Part of the Chemical Engineering Commons

\footnotetext{
Pattanayak, Abhinandan and Subramanian, Anuradha, "Impact of Porogens on the Pore Characteristics of Zirconia Particles Made by Polymer-Induced Colloid Aggregation" (2011). Anuradha Subramanian Publications. 9.

https://digitalcommons.unl.edu/cbmesubramanian/9
}

This Article is brought to you for free and open access by the Chemical and Biomolecular Research Papers - Faculty Authors Series at DigitalCommons@University of Nebraska - Lincoln. It has been accepted for inclusion in Anuradha Subramanian Publications by an authorized administrator of DigitalCommons@University of Nebraska - Lincoln. 
Published in International Journal of Applied Ceramic Technology 8:1 (January/February 2011), pp. 94-111;

doi: 10.1111/j.1744-7402.2009.02410.x Copyright (C) 2009 American Ceramic Society; published by John Wiley \& Sons.

Used by permission. http://onlinelibrary.wiley.com/doi/10.1111/j.1744-7402.2009.02410.x/abstract

Published online August 3, 2009.

This work was supported through a grant from the National Science Foundation (CTS 0411632).

\title{
Impact of Porogens on the Pore Characteristics of Zirconia Particles Made by Polymer-Induced Colloid Aggregation
}

\author{
Abhinandan Pattanayak and Anuradha Subramanian \\ Department of Chemical and Biomolecular Engineering, \\ University of Nebraska-Lincoln, Lincoln, Nebraska 68588 \\ Corresponding author - A. Pattanayak, email abhi.pattanayak@gmail.com
}

\begin{abstract}
Zirconia particles were prepared from a $20 \%$ colloidal sol $\left(\mathrm{ZrO}_{2}\right)$ by the polymer-induced colloid aggregation (PICA) process, both in the presence and absence of porogens. Specifically, porous zirconia particles having varying porosity were prepared by a two-step protocol wherein a porogen was first embedded during the particle synthesis, followed by its removal in a subsequent step. In this research study, the ability of four different types of porogens, viz. fumed silica, micronized high molecular weight polyethylene emulsion ME09730, and microcrystalline waxes ME48040M2 and ME98040M1, to impact the pore size, porosity, and pore area were investigated. Particle morphology and porosity of the resultant particles were characterized by scanning electron microscopy, mercury intrusion-extrusion porosimetry, and the nitrogen adsorption-desorption sorptometry. Optimal ratios of zirconia sol and porogen to yield the largest unimodal distribution of pores were determined. Porous zirconia particles were obtained after the removal of porogen, followed by a calcination and sintering protocol. Particle aggregates were typically 7-45 $\mu \mathrm{m}$ in diameter, with BET surface areas between 21 and $54 \mathrm{~m}^{2} / \mathrm{g}$ and pores ranging from 18 to $120 \mathrm{~nm}$ in diameter. The nitrogen sorptometry and mercury porosimetry data of these support particles were modeled to calculate surface fractal dimension, pore accessibility parameters, pore network, and pore geometry.
\end{abstract}

\section{Introduction}

The popularity of chromatographic processes in bioseparations has grown with advances in support materials and their fabrication, resulting in increased resolution in less time. ${ }^{1}$ Supports based on zirconia, particles with high density and excellent thermal and chemical stability, can offer increased flexibility relative to silica and polymeric phases when designing separations. While the surface chemistry of zirconia has been stud- ied in detail, the widespread applicability of zirconia particles in chromatography is precluded by the unavailability of zirconia particles with varying particle and pore sizes. Based on prior work, which has enabled the preparation of porous zirconia particles by polymerinduced colloid aggregation (PICA) $)^{2,3}$ and spray drying of colloidal zirconia suspension and has documented its use as a stable chromatographic support ${ }^{4}$ the objective of this study is to further optimize the PICA process to produce particles and monoliths with varying sizes and 
a controlled and hierarchical pore architecture. Our results have also highlighted the need to further optimize the surface area, pore size, and pore volume for the retention and separation of biologically relevant biomolecules, as we have found that the transport of biomolecules in the zirconia particles with a pore size of $22 \mathrm{~nm}$ is limited by pore diffusion. In addition, calculations based on Renkin's equation show that for separations of biomolecules of sizes ranging from 8 to $15 \mathrm{~nm}$, supports must have pore sizes in the range of $40-75 \mathrm{~nm}$ for optimal performance, that is the pore diameter must be greater or equal to five times the size of the molecules being transported. The next logical step is to produce zirconia supports with particle diameters in the range of $50-200 \mu \mathrm{m}$ and with pore sizes in the range of 35-100 nm.

Wet chemistry techniques such as the sol-gel and surfactant emulsion processes have been combined with organic and inorganic templates to fabricate macro/mesoporous structures of oxides like silica, titania, and zirconia. Successful and selective removal of these templates replaces the template sites with hollowness. ${ }^{5}$ In an attempt to control the pore diameters and pore volumes, the zirconia particles were first prepared by the sol-gel process ${ }^{6}$ and then incubated in a solution containing $\mathrm{NaCl}$, the porogen, 7,8 in order to prevent the collapse of the pore structure during the essential sintering step. Zirconia particles with a particle size of $1.3 \pm .5 \mu \mathrm{m}$ and pore size $20-35 \mathrm{~nm}$ were obtained. The literature cites the use of diverse templates such as latex, ${ }^{9}$ cellulose acetate, cellulose nitrate, polyamide, polyethersulfone, polypropylene, ${ }^{10}$ acryl amide, glycidylmethacrylate, ${ }^{11}$ polystyrene cross-linked with divinyl benzene (beads), ${ }^{12}$ polymethylmethacrylate, $^{13}$ and so forth. The overall outcome of this category revealed particles of size $0.1-2.5 \mu \mathrm{m}$, pore size $2.5-700 \mathrm{~nm}$, and surface area 25 $600 \mathrm{~m}^{2} / \mathrm{g}$, depending on the precursor and template being used. Significant progress has been made in understanding and formulating routes to produce hollow spheres (2-5 $\mu \mathrm{m}$ spheres, pore size $6-18 \mathrm{~nm}$ ) of crystalline porous metal oxides by nanocasting with leachable mesoporous hollow shells. ${ }^{14,15}$ Furthermore, it was proposed that ultrasonic cavitation during solgel reactions ${ }^{16}$ and high-frequency microwave sintering 17 improved the particle morphology. In addition, immense research has been conducted with popular surfactants like cationic dodecyltrimethylammoniumbromide and cetyltrimethylammoniumbromide, anionic sodium dodecylsulfate, ${ }^{18,19}$ nonionic polyethyl- ene oxide, and poly(ethylene oxide)-poly(propylene oxide)-poly(ethylene oxide) block copolymer. ${ }^{20,21}$ Zirconia powders synthesized using macroemulsion and microemulsion techniques, involving mixing a $\mathrm{Zr}^{4+}$ emulsion with aqueous ammonia droplets to precipitate $\mathrm{ZrO}_{2}{ }^{\prime 2,23}$ produced $0.3-3 \mu \mathrm{m}$ diameter particles.

In summary, our survey of the literature reveals few studies that address the synthesis of stable porous zirconia particles in the size range of $10-200 \mu \mathrm{m}$ with a controlled pore architecture. In this study, we have judiciously attempted to manipulate and optimize the pore architecture of zirconia particles by impregnating them with a leachable porogen during the particle aggregation step. We hypothesize that the removal of these porogens in a subsequent step will produce particles with larger pore diameters. We have used the PICA method for aggregating zirconia colloid, as the PICA method optimally produces particles of strict and distinct size ranges. ${ }^{2,3}$ We have used fumed silica, cationic polyethylene (ME09730), and nonionic microcrystalline wax (MCW) emulsions (ME48040M2 and ME98040M1) as putative porogens. Zirconia particles produced by the PICA method have been characterized by BET nitrogen porosimetry, mercury porosimetry, and scanning electron microscopy (SEM). Also, this paper includes the application of a pore network model on the nitrogen sorption and mercury porosimetry data in order to investigate several crucial pore geometry-related parameters.

\section{Materials and Methods}

\section{Materials}

All reagents obtained from commercial sources were of analytical grades or better, and were used without further purification unless otherwise mentioned. Sodium hydroxide pellets, isopropyl alcohol, nitric acid, hydrochloric acid, formaldehyde (37\%), and granulated urea were purchased from Fisher Scientific (Hanover Park, IL). Aqueous zirconia sol ( $\mathrm{pH}=3.0)$, containing $20 \mathrm{wt} \%$ of $100 \mathrm{~nm}$ colloid of $\mathrm{ZrO}_{2}$, was purchased from Nyacol Products (Ashland, MA). Untreated fumed silica (CAB-O-SIL) was obtained from Cabot (Tuscola, IL). Ethylene glycol (99\%) was purchased from Acros Organics (Morris Plains, $\mathrm{NJ}$ ), and nonionic MCW emulsions (ME48040M2 and ME98040M1) and micronized cationic polyethylene emulsion (ME09730) were generous gifts from Michel- 
man (Cincinnati, OH). Potassium bromide (KBr) FTIR grade ( $>99 \%$ purity) was purchased from Sigma-Aldrich (St. Louis, MO).

\section{Methods}

Preparation of Particles by the PICA Method: Zirconia particles were prepared by the PICA method as detailed elsewhere. ${ }^{2,24}$ Briefly, $50 \mathrm{~mL}$ of presonicated zirconia sol, at a $\mathrm{pH}$ of 1.5 , was mixed well with $3.75 \mathrm{~g}$ urea. $6.25 \mathrm{~mL}$ of $37 \%$ formaldehyde solution was added and mixed for an additional minute..$^{25}$ After $2 \mathrm{~h}$ of polymerization, the reaction was quenched with $300 \mathrm{~mL}$ or a sixfold amount of DI water. ${ }^{25}$ The micrometer scale aggregates of colloids were separated by batch centrifugation at $1500 \mathrm{rpm}$ for $12 \mathrm{~min}$. Precipitated aggregates were resuspended in 2-propanol, collected, and dried in a vacuum oven at $90^{\circ} \mathrm{C} / 12 \mathrm{~h}$ and $175^{\circ} \mathrm{C} / 16 \mathrm{~h}$. Further heat treatment was comprised of calcination and sintering in a muffle furnace at $375^{\circ} \mathrm{C} / 2 \mathrm{~h}, 750^{\circ} \mathrm{C} / 6 \mathrm{~h}$, and $900^{\circ} \mathrm{C} / 3 \mathrm{~h}$. A $20^{\circ} \mathrm{C} / \mathrm{min}$ ramp was used between $375^{\circ} \mathrm{C}$ and $750^{\circ} \mathrm{C}$, while $40^{\circ} \mathrm{C}$ / min was used to reach $900^{\circ} \mathrm{C}$. The final product was cooled to room temperature, weighed, and stored in air-tight plastic sample containers.

PICA Particles with Porogens: Experiments were designed to synthesize zirconia particles using a variety of porogens (Table I) and at various porogen loadings. Table I lists the physical and chemical properties and nomenclatures used for easy referencing of these porogens.

PICA Particles with Fumed Silica: The commercially available sol, which is nominally at $20 \%$, was concentrated by centrifugation to obtain a $40 \%$ sol. When using fumed silica as a porogen, an acidic solution of fumed silica was added to a $40 \%$ zirconia sol. For example, for producing a 4:1 molar ratio of zirconia to fumed silica, $2.97 \mathrm{~g}$ of fumed silica was dispersed in $50 \mathrm{~mL}(\mathrm{pH}=1.5)$ of acidulated DI water for $30 \mathrm{~min}$. The resulting fumed silica solution was mixed vigorously with the $50 \mathrm{~mL}$ of presonicated $40 \%$ zirconia sol. $7.5 \mathrm{~g}$ urea and $12.5 \mathrm{~mL}$ formaldehyde were then added, and the remainder of the particle separation steps and the calcination were followed, similar to the general PICA described earlier. Fumed, silica laden, sintered PICA particles were added to a $3 \mathrm{M} \mathrm{NaOH}$ solution, and the mixture was either stirred on a heater-cum-stirrer plate with the temperature set at $80^{\circ} \mathrm{C}$ or heated in a muffle furnace at $95^{\circ} \mathrm{C}$ for $8-10 \mathrm{~h}^{26}$ with intermittent mechanical agitation or ultra-sonication. The leaching process was repeated three times with a fresh volume of $\mathrm{NaOH}$. In the final step, the particles were extensively washed with DI water, recovered by centrifugation, resuspended in 2-propanol, collected in shallow dishes, and subjected to heating, as described earlier.

PICA Particles with MCW and Polyethylene Emulsion: Porous zirconia particles were prepared with ME48040M2, ME98040M1, and ME09730 as porogens for zirconia:porogen molar ratios of 4:1, 2:1, and 1:1. Porogen amounts were calculated assuming an average of $590 \mathrm{~g} / \mathrm{mol}$ and $40 \%(\mathrm{w} / \mathrm{w})$ of nonvolatile components. In each of these experiments, $50 \mathrm{~mL}$ of $20 \%$ sol at $\mathrm{pH} 1.5$ was taken in a $500 \mathrm{~mL}$ beaker and sonicated for $10 \mathrm{~min}$. A $9.7 \mathrm{ml}$ emulsion (corresponding to a $4: 1$ ratio) was then added to the sol and the $\mathrm{pH}$ was re-adjusted to 1.5 using concentrated nitric acid. After stirring vigorously for 15-20 minutes, $4.5 \mathrm{~g}$ of urea was added and the mixture was stirred for 30 minutes to allow nucleation. Finally, $7.3 \mathrm{ml}$ of $37 \%$ formaldehyde was added and the solution was stirred for an additional minute. The remaining steps of reaction quenching, processing, and separation of the aggregates were followed similar to the basic PICA particle making protocols. The molar concentrations of urea and formaldehyde in the overall solution were independent of the total volume of zirconia and wax emulsion mixture and were always maintained at 1.25 and $2.4 \mathrm{M}$. The wax-based porogens were removed by heating. The

Table I. Porogen Nomenclature and Physical/Chemical Characteristics

\begin{tabular}{lllclcr}
\hline Symbol & Name & $\begin{array}{l}\text { Physical } \\
\text { state }\end{array}$ & $\begin{array}{l}\text { Melting } \\
\text { point }\left({ }^{\circ} \mathrm{C}\right)\end{array}$ & $\begin{array}{l}\text { Chemical } \\
\text { nature }\end{array}$ & $\begin{array}{c}\mathrm{pH} \\
\begin{array}{c}\text { Particle } \\
\text { diameter (nm) }\end{array}\end{array}$ \\
\hline P1 & Fumed silica & Solid powder & - & Inorganic & $<6(2.3$ iep) & $\sim 150-175$ \\
P2 & ME48040M2 & Liquid & $80-85$ & Organic/synthetic & $1-12$ & 300 \\
P3 & ME98040M1 & Liquid & $80-85$ & Organic/synthetic & $2-12$ & 700 \\
P4 & ME09730 & Liquid & $130-145$ & Organic/synthetic & $<7$ & 150 \\
\hline
\end{tabular}


PICA heat treatment protocol was modified slightly by allowing a slower rate of increase of the temperature to $375^{\circ} \mathrm{C}$, with $10^{\circ} \mathrm{C} / \mathrm{min}$ as the ramp between $175^{\circ} \mathrm{C}$ and $375^{\circ} \mathrm{C}$, and $5^{\circ} \mathrm{C} / \mathrm{min}$ over reaching to $175^{\circ} \mathrm{C}$ from $100^{\circ} \mathrm{C}$.

\section{Material Characterization}

\section{FTIR Spectrometry}

FTIR grade ( $>99 \%$ purity) $\mathrm{KBr}$ was used to prepare the pellets. IR spectra analysis was performed on a Nicolet 510P FTIR spectrometer (Nicolet Instrument, Madison, WI) equipped with dry air at $10 \mathrm{psi}$, for wavenumbers from 400 to $4000 \mathrm{~cm}$, with a resolution of $4 \mathrm{~cm}$. The percentage of average transmittance was recorded for 32 scans for each sample tested.

\section{SEM Imaging}

SEM imaging was carried out with a Hitachi S7400 (Tokyo, Japan) on all the PICA samples (electron microscopy facility, University of Nebraska-Lincoln) for accelerating voltages between 10 and $25 \mathrm{kV}$. Photographs were taken of a representative sample of each powder by sparingly sprinkling the powder onto double-sided sticky carbon conductive tape (Ted Pella, Redding, CA) that was attached to an SEM stub. The particles were then coated with a chromium film of the thickness $0.2 \mathrm{~nm}$ by DC sputtering at $125 \mathrm{~mA}$ for $60 \mathrm{~s}$. The backscattered electron images of the particles produced at the accelerating field were acquired as grayscale images.

\section{PSD/TSD from $\mathrm{N}_{2}$ Sorption}

PSD/TSD from nitrogen adsorption and desorption isotherm data at $77 \mathrm{~K}$ were obtained using a Micromeritics ASAP 2010 sorptometer (Norcross, GA). About $250 \mathrm{mg}$ of each sample was analyzed at 42 different $\mathrm{P} / \mathrm{P}_{0}$ (relative pressure) points on both the adsorption and desorption branches. The relative pressure points of analysis were deliberately sparsely spaced at lower $\mathrm{P} / \mathrm{P}_{0}$ values and densely populated at higher values to enable a detailed PSD curve for the larger pore size regime. The isotherms obtained from a plot of volume adsorbed $\left(\mathrm{cm}^{3} / \mathrm{g} \mathrm{STP}\right)$ versus relative pressure $(\mathrm{P} /$ $\mathrm{P}_{0}$ ) were compared against the following: (1) a Type-
IV isotherm with an H1 hysteresis loop (indicates the presence of "cylindrical" pores), (2) a Type-IV isotherm with an $\mathrm{H} 2$ hysteresis loop (indicates the presence of "ink-bottle shaped" pores), and (3) a Type-IV isotherm with an intermediate-type $\mathrm{H} 1-\mathrm{H} 2$ hysteresis loop (indicates a combination of "ink-bottle shaped" and "cylindrical" pores). ${ }^{7,8}$

\section{PSD/TSD from Mercury Porosimetry}

Mercury porosimetry was performed for select samples presented in this paper. All the samples were sent for analysis to the Particle Engineering Research Center at the University of Florida. A Quantachrome Autoscan 60 Mercury Porosimeter (Boynton Beach, FL) was used to analyze the samples. Quantachrome Autoscan Poro2PC (Version 3.0) software was used to generate pore-related data. Each analysis required about $1 \mathrm{~cm}^{3}$ of sample, and the pore size calculations and surface areas were based on a contact angle of $140^{\circ}$.

\section{Results and Discussion}

Our research indicates that the performance of the first generation of zirconia particles produced by the PICA process, which yielded a particle size of 1-3 $\mu \mathrm{m}$ and a pore size of 18-24 nm, or the spray drying process, which yielded a particle size of $25-38 \mu \mathrm{m}$ and pore size of $18-24 \mathrm{~nm}$, is limited by pore diffusion. ${ }^{27-30}$ Such findings have also been observed in previous work with porous supports. ${ }^{6,31,32}$ An examination of the relationship between the size of the colloid and the pore size obtained in aggregates obtained by the spray drying process, the PICA process, or the oil-emulsion process reveals that when a colloidal solution (with the colloid particle size of $100 \mathrm{~nm}(1000 \AA)$ ) was used in any of the aggregation processes listed, a pore size in the range of 18-27 $\mathrm{nm}$ was often obtained. ${ }^{3,33-35} \mathrm{In}$ the absence of porogen or any binder molecule, we hypothesize that the most viable arrangement of colloid particles is one in which three colloid particles are held in the cluster. The resultant pore diameter obtained equals the equivalent diameter of the interstitial void space between colloid particles. Mathematically, this can now be approximated as follows: $D_{p}=0.227 D_{c^{\prime}}$ where $D_{p}$ is the effective pore diameter obtained, and $\mathrm{D}_{\mathrm{c}}$ is the diameter of the colloid particle. We have used a colloid in which the particles are $100 \mathrm{~nm}$ in diame- 
ter; thus, the theoretical pore size one could obtain in the absence of a porogen is $22.7 \mathrm{~nm}$. We have confirmed this by experimentation, and thus it is not surprising that both the PICA and SD methods resulted in similar pore sizes, as they both used a $100 \mathrm{~nm}$ colloid. An alternative to the current approach, which uses a $100 \mathrm{~nm}$ colloid, would be to use colloidal zirconia with larger sizes (i.e., $200-400 \mathrm{~nm}$ ) to generate particles with greater pore sizes. However, it is worth noting that current techniques to produce zirconia colloid with larger sizes are tedious, cumbersome, uneconomical, and larger size colloidal zirconia is not commercially available. In addition, attempts to generate $2000 \AA(200 \mathrm{~nm})$ and $4000 \AA$ (400 nm) zirconia colloid were unsuccessful (A. Subramanian and P. W. Carr, unpublished results). Thus, in this report, leachable porogens were included in the colloid before the initiation of the particle aggregation step, and we have used porogens with similar or larger dimensions to the final desired pore sizes, 50-100 $\mathrm{nm}$. We have repeated all the experiments in triplicate for each molar ratio of zirconia sol and porogen pertaining to different porogen categories. BET was obtained for all the samples. BETs for a particular ratio were similar, and only the representative BET has been reported.

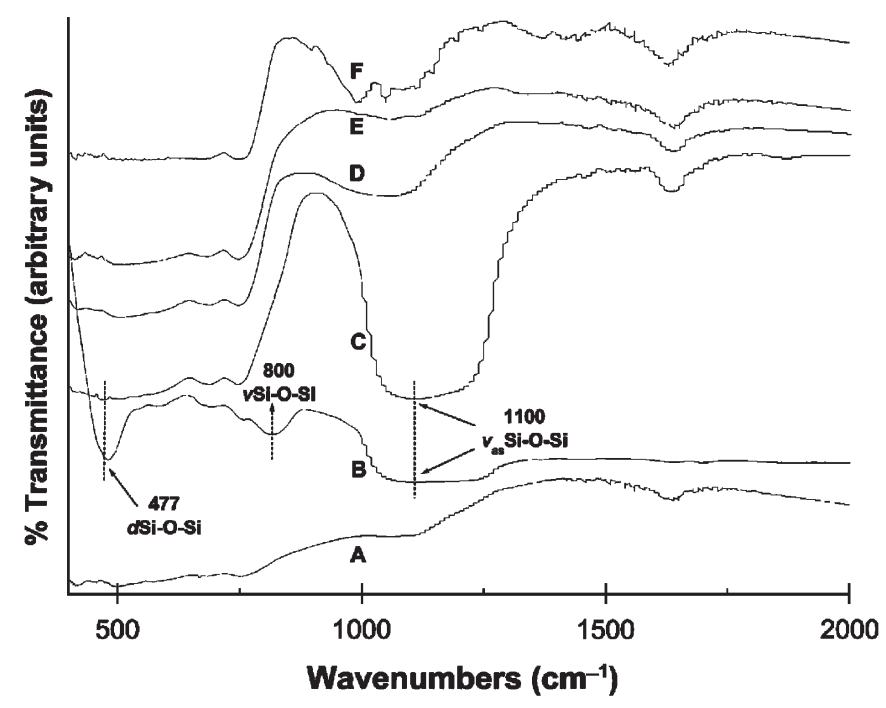

Figure 1. FT-IR spectra of (A) pure zirconia, (B) pure fumed silica, (C) polymer-induced colloid aggregation (PICA) particles containing fumed silica in the zirconia:fumed silica molar ratio of $4: 1$, (D) the PICA particles treated thrice with $3 \mathrm{M}$ $\mathrm{NaOH}$ solution, (E) the PICA particles treated with $4.95 \% \mathrm{HF}$ solution, and $(\mathrm{F})$ the PICA particles treated with ethylene-glycol-NaOH solution.

\section{FTIR Spectrometry}

Figure 1 shows a comparative study of infrared spectra of six samples, viz (A) pure zirconia calcined at $900^{\circ} \mathrm{C}$ for $3 \mathrm{~h},(\mathrm{~B})$ pure fumed silica, (C) PICA particles prepared from a 4:1 molar ratio of zirconia sol and fumed silica, (D) the PICA particles treated thrice with a $3 \mathrm{M} \mathrm{NaOH}$ solution, (E) the PICA particles treated with a $4.95 \%$ HF solution, and (F) the PICA particles treated with an ethylene-glycol- $\mathrm{NaOH}$ solution, respectively. As is evident from spectrum B, the typical band of pure fumed silica at $800 \mathrm{~cm}$ was ascribed to the symmetric stretching of oxygen in the $\mathrm{Si}-\mathrm{O}-\mathrm{Si}$ group. The broad band at $1100 \mathrm{~cm}$ is also evident, which was assigned to the $\mathrm{v}_{\text {as }}(\mathrm{Si}-\mathrm{O}-\mathrm{Si})$ asymmetric stretching of the siloxane group. In addition, a band around 450, $477 \mathrm{~cm}$ to be precise, was also detected, which was ascribed to the rocking vibrations of the $\mathrm{Si}-\mathrm{O}-\mathrm{Si}$ group. In consonance with the literature, ${ }^{36}$ the strongest modes, as a result of asymmetric stretching in transnuclear siloxane rings, occurred in the frequency range of 950-1250 cm. However, in the calcined sample, spectrum C, only the silica bands at 1100 and $477 \mathrm{~cm}$ were present. The frequencies characteristic of fumed silica were absent in spectra D, E, and F, confirming its complete removal after the specific treatments.

\section{Microscopy}

PICA Particles Made Using Fumed Silica: In Figure 2, typical SEM images of (a) PICA particles prepared with zirconia and porogen P1 (fumed silica), and (b) particles after the porogen-leaching step have been shown. It was observed that at low porogen loading, the primary particles were uniform spheres, with diameters ranging from 1.5 to $2.5 \mu \mathrm{m}$, which subsequently reduced in size at higher loading. The secondary particles were orderly arranged clusters of the primary spheres of sizes 20-35 $\mu \mathrm{m}$. Orderliness was disrupted at a higher fumed silica concentration, and the aggregates were nebulous. Particle sphericity was completely lost for the aggregates that contained higher amounts of fumed silica. The morphology after the removal of the porogen presented a complicated arrangement of the zirconia colloids.

PICA Particles Made Using Polymeric Porogens: The addition of polymeric porogens (P2, P3, and P4) revealed a different class of morphology, where surface imaging alone posed a difficulty in understanding the effects of increasing porogen content in the starting 

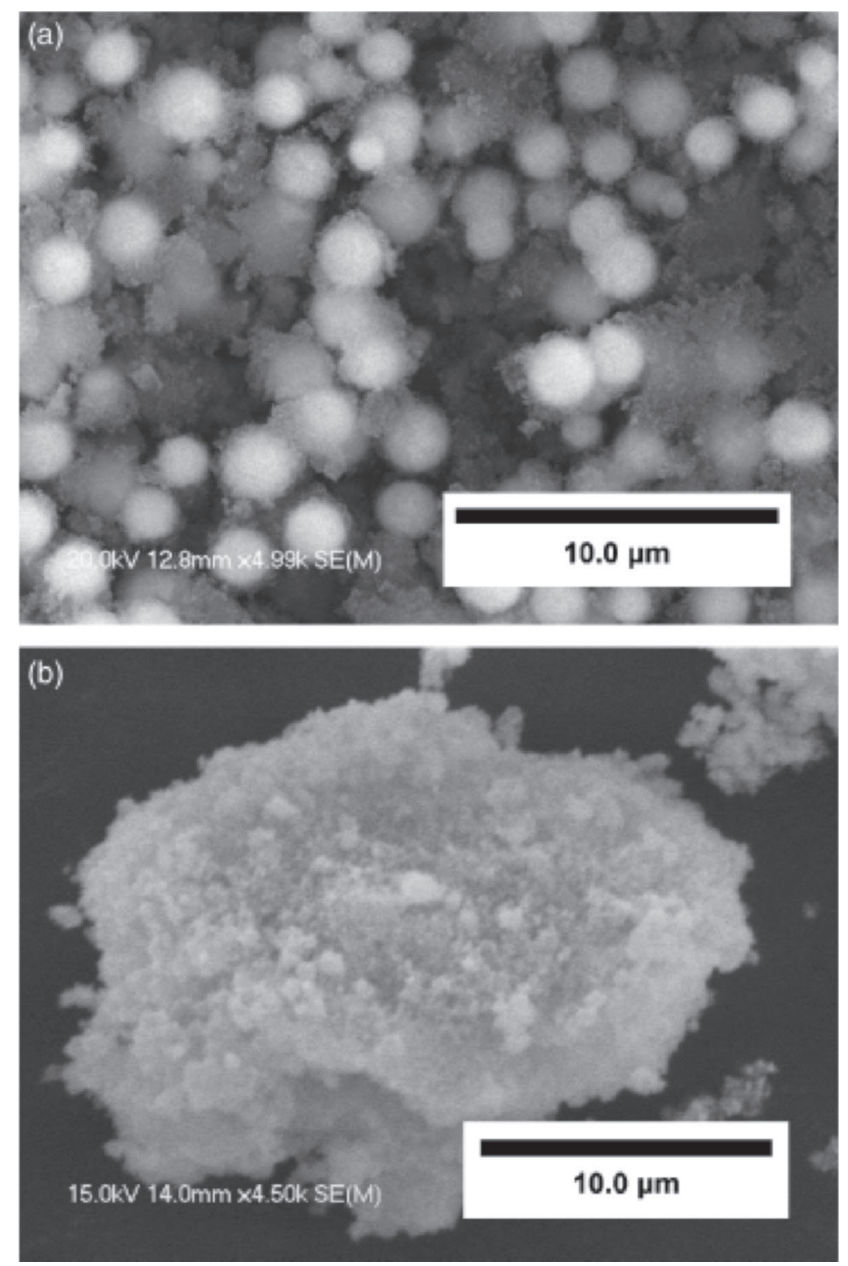

Figure 2. Representative scanning electron microscopic images of zirconia aggregates prepared from porogen P1 (fumed silica) with $40 \%$ zirconia sol. (a) Shows aggregate morphology after porogen addition; (b) shows morphology after leaching of fumed silica using $3 \mathrm{M} \mathrm{NaOH}$.

zirconia sol mixture on the final sintered particles. Combined SEM images of this genre of particles at various magnifications have been shown in Figures 3ad. At very low ratios of zirconia and porogen (8:1 and 6:1), the aggregates showed evidence of the formation of distorted spherical particles (SEM not shown in the results), which is a characteristic of PICA experiments. However, for higher ratios like 4:1, 2:1, and 1:1, SEM imaging was not very effective in distinguishing the changes in the morphologies. In general, all the higher porogen ratios showed sintered aggregates with sizes ranging from four to several hundred micrometers. There was random distribution of both the shape and the size of these particles. The surface of the secondary aggregates frequently showed a continuous distribution of deep recesses and surface cracks. In addi- tion, subsurface tunnels and their interconnectedness were evident, as shown in some of the images taken at higher magnifications. Often, the widths of the surface recesses were observed to have dimensions similar to those of the porogens.

\section{Effect of Porogens and their Concentrations}

Zirconia with Porogen P1, Fumed Silica: Figure 4 shows a plot of nitrogen sorption isotherms for porous aggregates prepared with porogen P1 (fumed silica) in varying ratios with zirconia sol. It is clear that the addition of porogen led to an increase in the total available volume of pores in the material. The heights of the isotherms showed an increasing trend with an increase in porogen content, although the ratio 4:1 was an exception, where the height of the isotherm was higher than the 2:1 ratio. The isotherms depict a Type-IV system with loops intermediate of $\mathrm{H} 1$ and $\mathrm{H} 3$, indicating that the pore paths might not be cylindrical in shape. The abrupt increase in adsorbed nitrogen volume toward the higher partial pressure indicates that the pores were large. This is confirmed by the pore size distribution, where the curves were incomplete due to the experimental measurement limitations of the sorption mechanism. Interestingly, irrespective of the increase of the height of the isotherms, that is the increase in the adsorbed volume of nitrogen, the psd did not show a shift of curves toward larger pore sizes for an increased porogen content. Even the throat size distribution was similar for the different ratios. This can only be possible when there is minimal intraporogen association to form larger porogen bodies, around which the zirconia colloid may associate and construct the pore wall. In this case, the porogens were distributed as individual entities and thereby offered a greater number of pores of the same size range throughout the sample.

Zirconia with Porogen P2, ME48040M2: Figure 5 shows the effect of concentration of the porogen P2 on sorption isotherms and pore/throat size distributions. It is clearly seen that increased porogen led to an increase in the height of the adsorption/desorption isotherms systematically, although there was not a significant difference among the peaks. In addition, the width of the hysteresis loop, which is predominantly Type-IV H1-H3, decreased and the isotherms shifted toward the higher partial pressure. This behavior signifies that the pores have been enlarged. The inset psd 

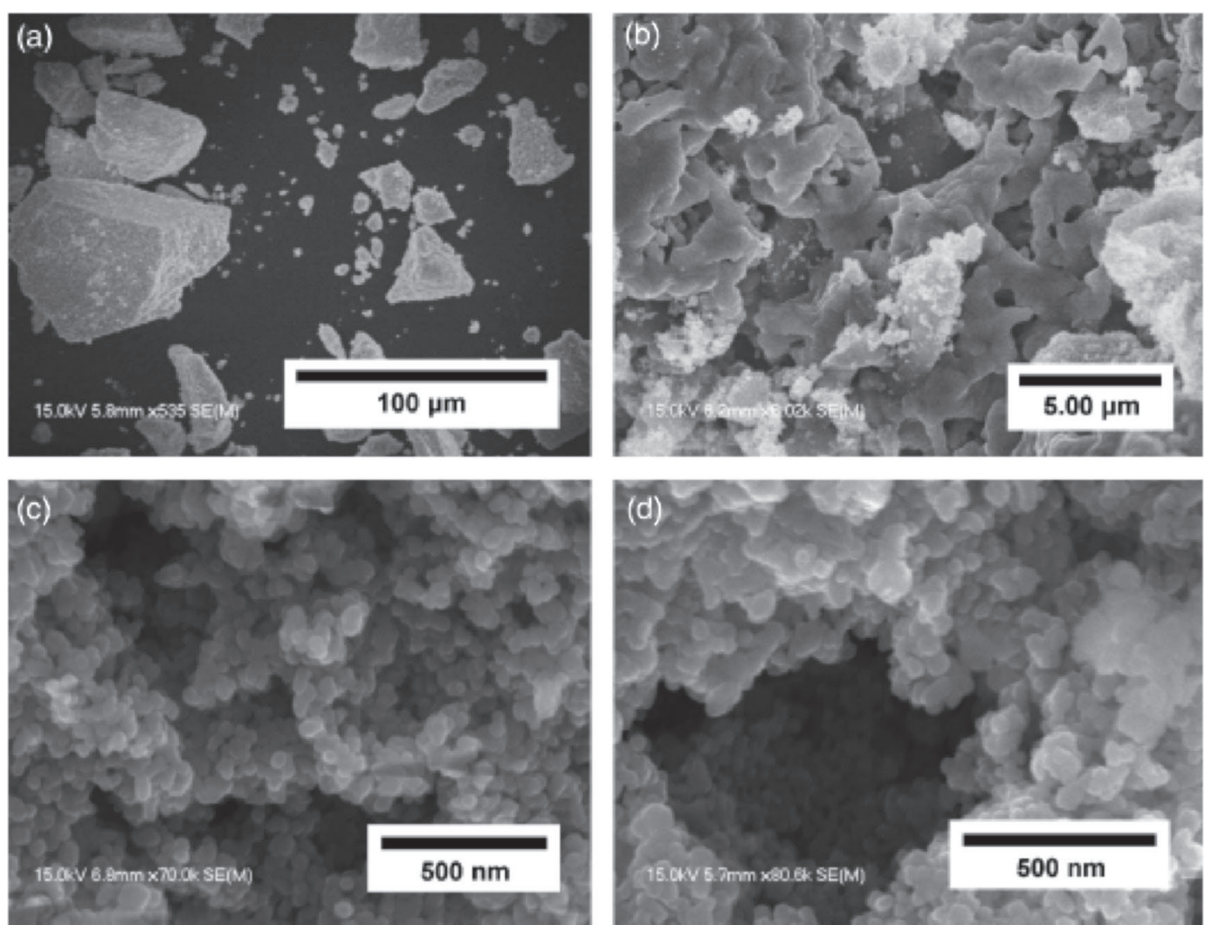

Figure 3. Scanning electron microscopic images a-d, at various magnifications, showing the typical genre of sintered zirconia aggregates prepared using polymeric porogens P2 (ME48040M2), P3 (ME98040M1), and P4 (ME09730).

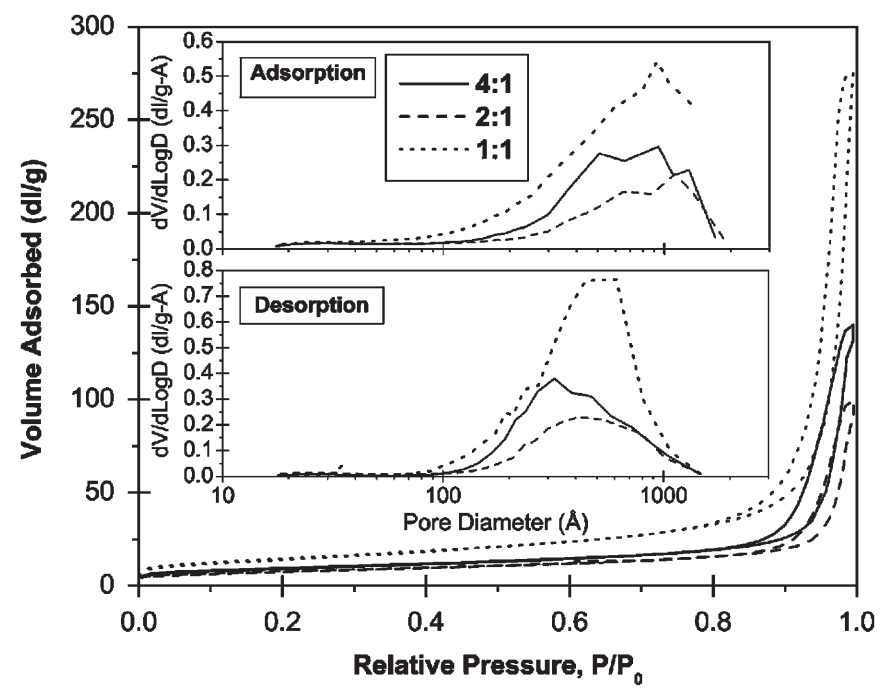

Figure 4. Adsorption/desorption isotherms and pore/throat volume distributions for sintered zirconia particles made using porogen P1 (fumed silica). Each ratio was repeated in triplicate. BETs for a ratio were similar, and a representative BET has been shown.

and tsd plots also confirm the increase in sizes of pores and throats. The heights of pore and throat peaks have continuously decreased for the increasing porogen content. Distinctively, the pore and throat peaks have shifted toward the right, with pore peaks at 45,75 , and

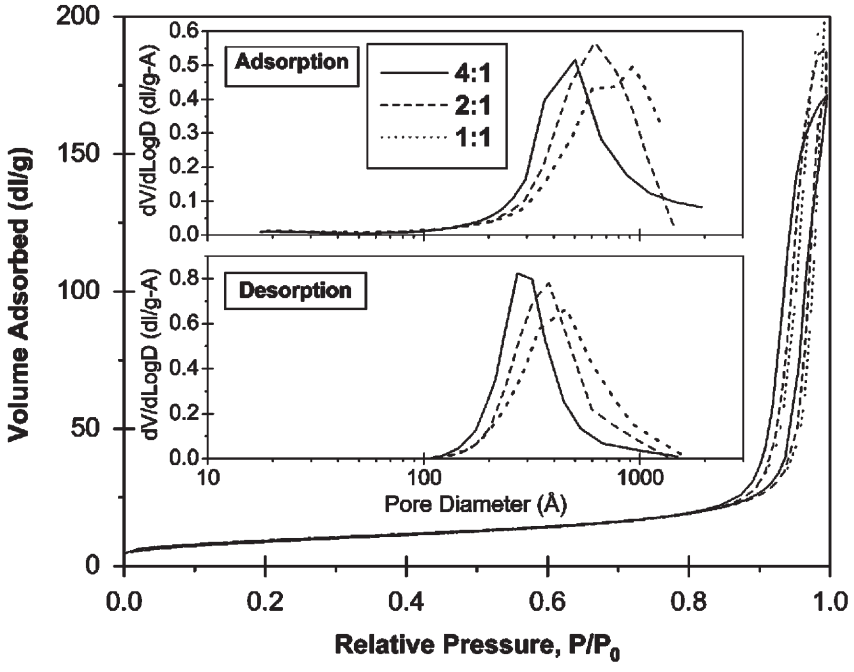

Figure 5. Adsorption/desorption isotherms and pore/throat volume distributions for sintered zirconia particles made using porogen P2 (ME48040M2). Each ratio was repeated in triplicate. BETs for a ratio were similar, and a representative BET has been shown.

$90 \mathrm{~nm}$ and throat peaks at 30, 37, and $42 \mathrm{~nm}$. Theoretically, the shifting trend may be attributed to an intraporogen association, around which colloid particles associate, and pore wall thinning, which were quite absent for the case with fumed silica. 


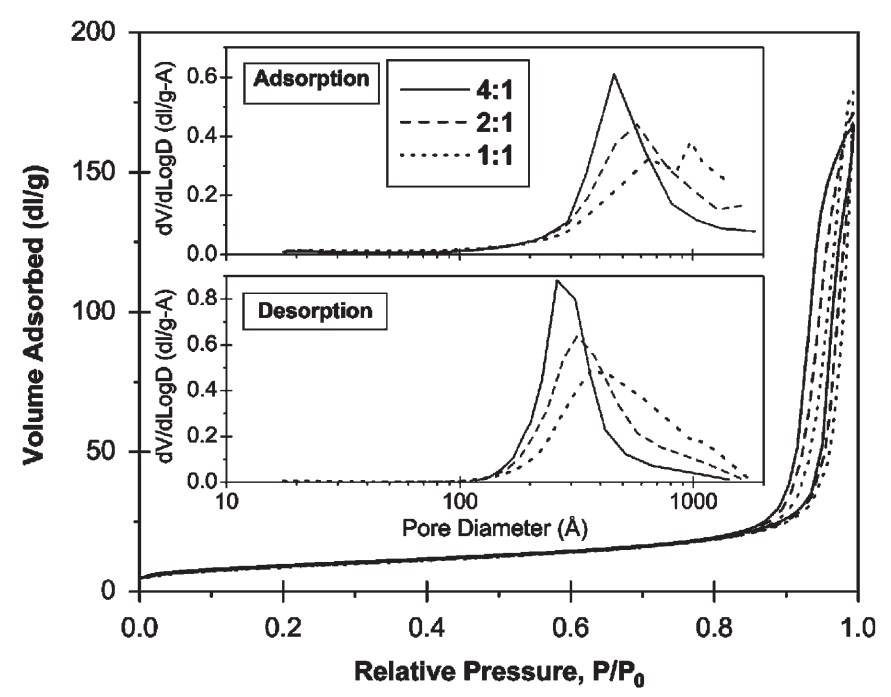

Figure 6. Adsorption/desorption isotherms and pore/throat volume distributions for sintered zirconia particles made using porogen P3 (ME98040M1). Each ratio was repeated in triplicate. BETs for a ratio were similar, and a representative BET has been shown.

Zirconia with Porogen P3, ME98040M1: Figure 6 shows nitrogen sorption results for zirconia particles prepared with porogen P3. The observations about the isotherms and the shift in pore and throat dimensions toward the higher range with increasing porogen loading may be explained in a similar manner as above, for porogen P2. The pore distribution curves calculated from the adsorption branch showed incomplete curves for pore sizes beyond $100 \mathrm{~nm}$, indicating the possibility of larger pores.

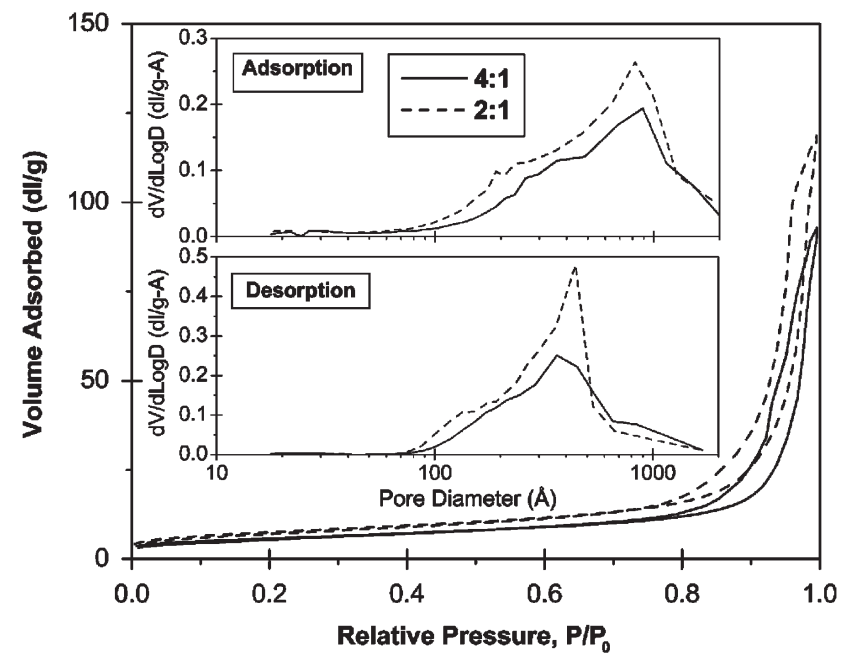

Figure 7. Adsorption/desorption isotherms and pore/throat volume distributions for sintered zirconia particles made using porogen P4 (ME09730). Each ratio was repeated in triplicate. BETs for a ratio were similar, and a representative BET has been shown.
Zirconia with Porogen P4, ME09730: Figure 7 shows adsorption/desorption isotherms and the calculated pore and throat size distributions for zirconia with porogen P4. The height of the isotherms increased with an increase in porogen loading. However, compared with the heights obtained for porogens P1, P2, and P3, these isotherms are significantly lower. This indicates that the contribution of pores in the range 10-100 nm was less. The isotherms are Type-IV, with abrupt termination at the higher partial pressure. The pore size distribution obtained from the adsorption isotherm is very broad and incomplete at the higher size range. The distributions also show that within the 10-100 $\mathrm{nm}$ range, the height of the curve increased with an increase in porogen loading, which signifies that although the basic pore size has not increased, the number of pores in that size class has increased.

\section{Comparative Analysis of the Porogens $-\mathrm{N}_{2}$ Sorption}

Figure 8 shows a comparative study of sorption isotherms and PSDs/TSDs. The samples selected for study had the optimum zirconia to porogen ratios for the respective porogens. Each of these ratios produced the highest isotherm in its porogen category. The isotherms suggest that porogen P1 had the highest peak with the possibility of micropores. The pore distribution was broad, starting from the $10 \mathrm{~nm}$ range. Porogen $\mathrm{P} 4$ produced the shortest isotherm. It had a broad and flatter pore size distribution in the $10-100 \mathrm{~nm}$

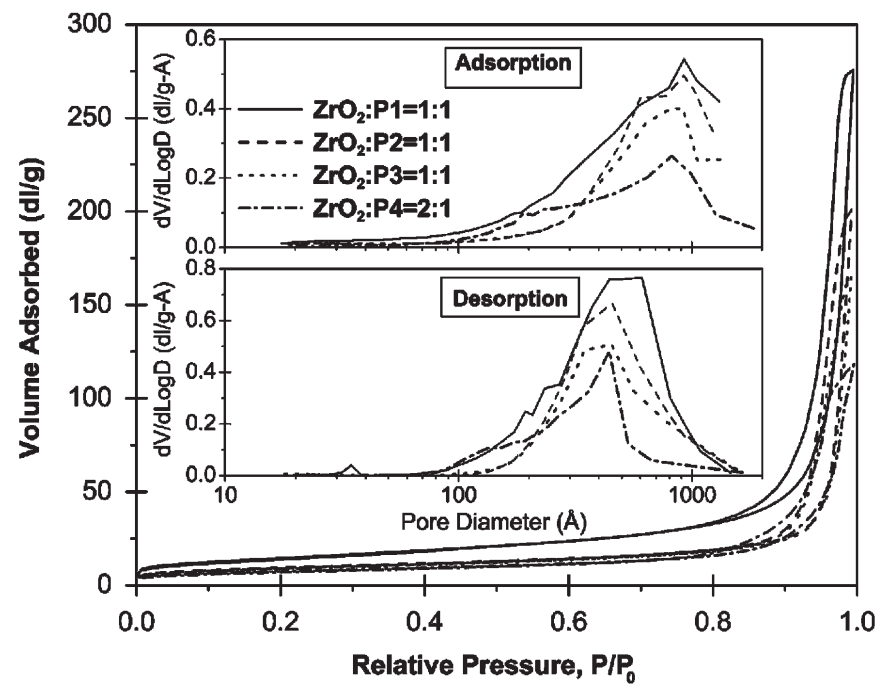

Figure 8. Comparative adsorption/desorption isotherms and pore/throat volume distributions for zirconia particles prepared from the four different porogens for some optimum ratios of zirconia sol and porogen. 
range. However, the throat size distribution was narrow. The other two porogens, P2 and P3, had characteristics intermediate of those obtained in the cases of $\mathrm{P} 1$ and P4. All four pore distribution curves were incomplete beyond the $110 \mathrm{~nm}$ size limit, indicating the presence of larger pore chambers, undetected by the present gas sorption mechanism. Table II presents various results from the $\mathrm{N}_{2}$ sorptometry and mercury porosimetry of the samples.

\section{Comparative Analysis of the Porogens-Mercury Porosimetry}

The incomplete pore size distributions obtained from the adsorption isotherms necessitated further analysis of the above-selected samples through mercury porosimetry. As is customary, the mercury intrusion process analogously depicts the nitrogen desorption, and the extrusion process resembles the adsorption process. Figures $9 \mathrm{a}$ and $\mathrm{b}$ respectively show the mercury extrusion and intrusion pictures. The intrusion process showed that all the samples had throat sizes between 20 and $90 \mathrm{~nm}$, except for the sample made using P4, which showed a bimodal distribution. The second peak occurred between 100 and $1000 \mathrm{~nm}$, with a peak at around $600 \mathrm{~nm}$. The extrusion process revealed that porogen $\mathrm{P} 1$ produced a close-spaced bimodal chamber size distribution with peaks, respectively, at 90 and $104 \mathrm{~nm}$. Porogens P2 and P3 both gave narrow uni-modal pore size distributions with peaks at 110 and $150 \mathrm{~nm}$, respectively. The porogen P4 produced a bimodal distribution having a narrow peak at $110 \mathrm{~nm}$ and a broad distribution between 600 and $3500 \mathrm{~nm}$, with a peak at $1070 \mathrm{~nm}$. Furthermore, it was observed that while porogen $\mathrm{P} 1$ predominantly produced smaller pores and throats, the porogens P2 and $\mathrm{P} 3$ produced larger ones, probably owing to the different sizes of these porogens. And finally, porogen P4 produced a hierarchical network having, distinctively, two size classes. The apparent shift of peaks toward

Table II. Nitrogen Sorptometry and Mercury Porosimetry Data of PICA Samples

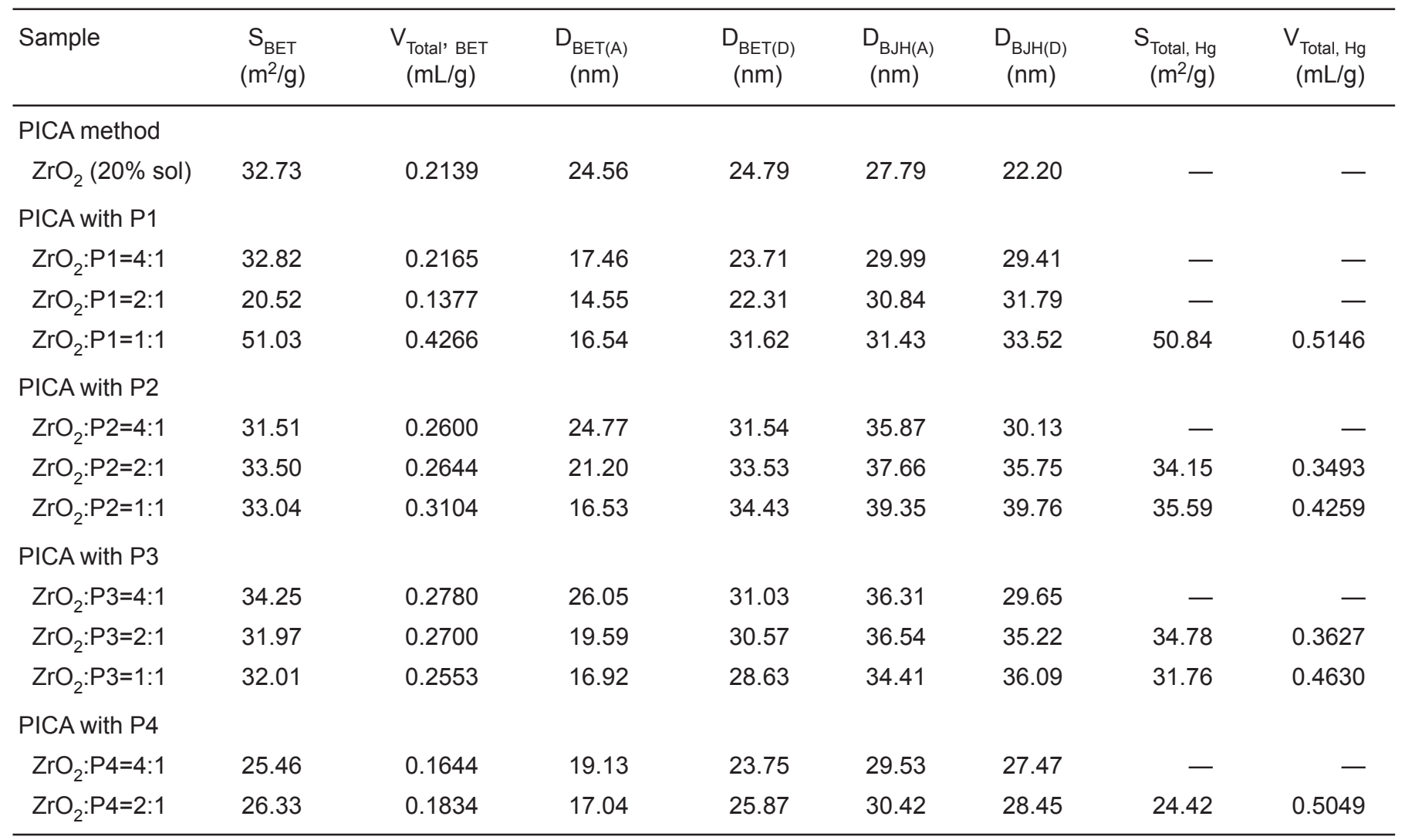

$\mathrm{S}_{\mathrm{BET}}$, multipoint BET surface area by nitrogen sorptometry; $V_{\text {Total, BET }}$, single point total pore volume of pores at $P / P_{O} \approx 0.995 ; D_{B E T(A)}$, average adsorption pore diameter calculated by the $B E T$ method at $P / P_{0} \approx 0.975 ; D_{B E T(D)}$, average desorption pore diameter calculated by the $B E T$ method at $\mathrm{P}_{\mathrm{P}} \approx 0.975 ; \mathrm{D}_{\mathrm{BJH}(\mathrm{A})}$, BJH method adsorption pore diameter; $\mathrm{D}_{\mathrm{BJH}(\mathrm{D})}$, BJH method desorption pore diameter; $\mathrm{S}_{\text {Total, Hg }}$, mercury intrusion surface area; $V_{\text {Total, } H g}$, mercury intrusion volume; PICA, polymer-induced colloid aggregation. 

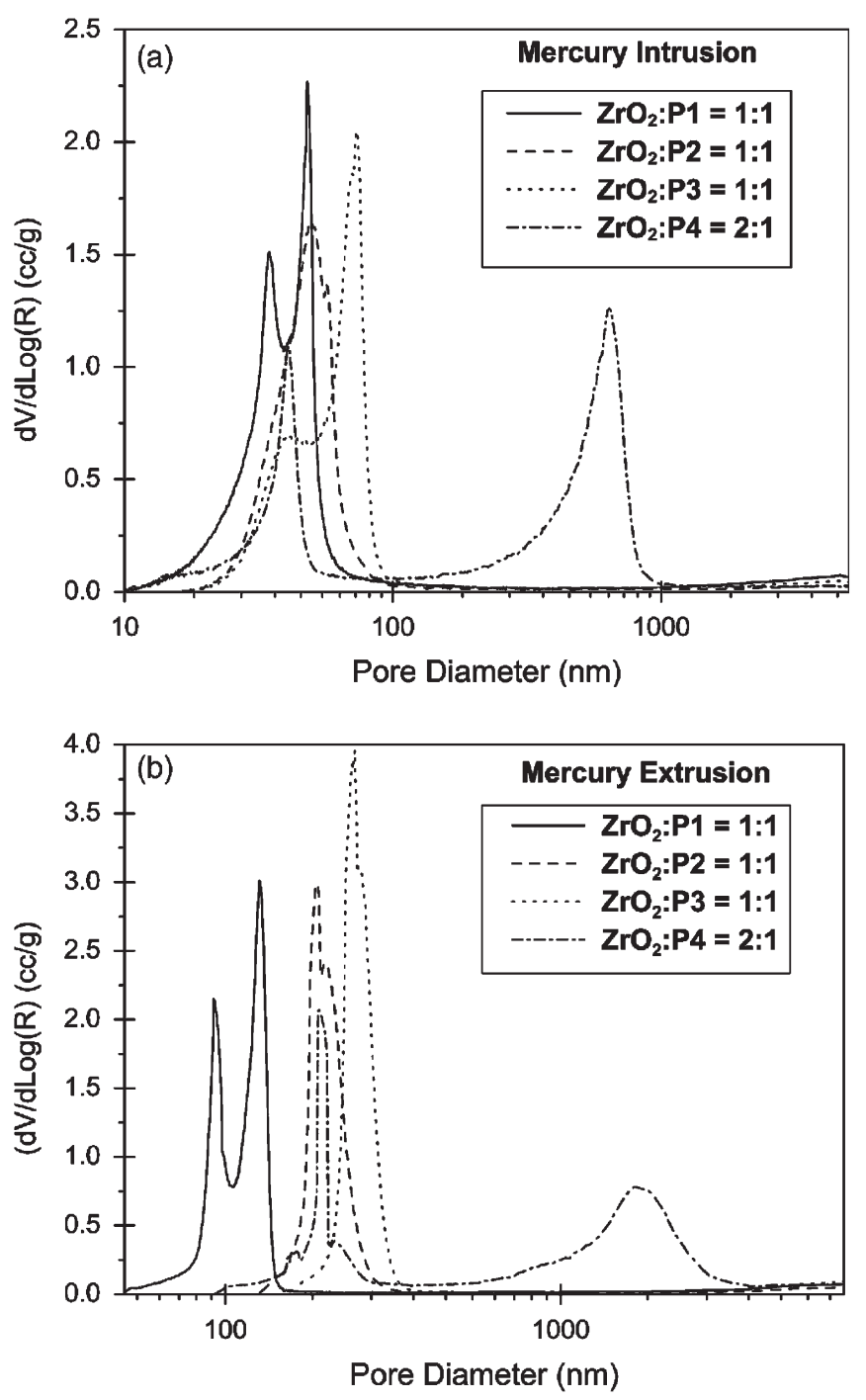

Figure 9. $(a, b)$ Mercury intrusion/extrusion results for polymer-induced colloid aggregation particles made from four different porogens.

higher sizes may be correlated with the size of the porogen used. While P1, P2, and P3 had nominal sizes of 175,300 , and $700 \mathrm{~nm}$, the shift in peaks can be substantiated based on the porogen size. However, the applicability of this thumb-rule breaks down while comparing the distributions for porogen $\mathrm{P} 4$, which has a particle diameter of just $150 \mathrm{~nm}$. Thus, other physical phenomena may have to be considered, for instance the intraporogen association around which colloids aggregate, pore structural deformation and pore body enlargement during porogen removal, and so forth. In addition, it may be hypothesized with confidence that the physico-chemical properties of the porogens also played a crucial part in imparting the final structure.
For instance, with reference to Table I, the very low melting points of porogens $\mathrm{P} 2$ and $\mathrm{P} 3$ made them amenable to structural deformation within, and also to the zirconia colloids surrounding them, at the very onset of the heat treatment. Any positive effects of intraporogen association were thus subdued and the pore sizes remained low. However, porogen $\mathrm{P} 3$ was at the advantage, owing to its higher melting point, and it provided enough support to the damp colloid structure during the initial drying phases.

\section{Model-Predicted Parameters}

The following subsections discuss the parameters obtained from the modeling of nitrogen adsorption/ desorption and mercury porosimetry data. All the parametric values and sample identifiers have been reported in Table III. Appendix A presents the rationale and application of the model in brief.

Sample A, PICA-20\%: Figure 10 shows experimental and model convergence curves for $\mathrm{N}_{2}$ adsorption/desorption data for PICA particles made from only $20 \%$ sol. From Table III, it is obvious that

- The high $C_{\mathrm{BET}}(=268)$ value indicates a significant presence of micropores; however, the $D_{\mathrm{s}}$ (2.0277) value close to 2 suggests a rather homogeneous material construction. The $\mathrm{s}$ value calculated by the relation $3 /\left(3-D_{s}\right)$ is 3.0854 .

- The sizes of throats $\left(\mu_{b}=3.9 \mathrm{~nm}\right)$ are clearly greater than the pores $\left(\mu_{\mathrm{s}}=3.1 \mathrm{~nm}\right)$, and the aspect ratio $\left(\mu_{\mathrm{s}} / \mu_{\mathrm{b}}=0.7843\right)$ indicates that the pore pathways are conical, or rather, ink-bottle shaped.

- The pores do not represent capillaries $\left(\beta_{\mathrm{s}}=1.64\right)$, and may be a slit type of high angular porosity $\left(n_{\mathrm{s}}=3.77\right)$.

- Very narrow pore $\left(\sigma_{\mathrm{s}} / \mu_{\mathrm{s}}=0.08\right)$ and throat $\left(\sigma_{\mathrm{b}} / \mu_{\mathrm{b}}=\right.$ $0.04)$ size distributions exist.

- The pore network is moderately connected $\left(\mathrm{q}_{\mathrm{bc} 0}=\right.$ 0.1778, $\left.\lambda_{\mathrm{bc} 0}=3.5736\right)$, and the Bethe coordination number is small $\left(Z_{\text {Bethe }}=6.6\right)$.

Sample B, PICA-P1-1:1: Figures 11a and b show convergence curves for PICA-P1-1:1. The pore and throat size distributions (see Figures 4 or 8 and 9) are unimodal, and no secondary drainage/desorption curves are present. The material is inhomogeneous, bearing rough surfaces and micropores. Throats are smaller than the pores, with narrow pore and throat size distributions. The pores do not represent capillaries and 
Table III. Model-Predicted Parameter Values and Sample Identifiers

\begin{tabular}{|c|c|c|c|c|c|}
\hline $\mathrm{C}_{\mathrm{BET}}$ & 267.94 & 284.97 & 283.10 & 275.02 & 294.39 \\
\hline $\mathrm{s}$ & 3.0854 & 3.9546 & 5.3927 & 3.1905 & 3.0886 \\
\hline$\mu_{S}(n m)$ & 3.1006 & 3.0668 & 3.1351 & 3.3186 & 1.6850 \\
\hline$\sigma_{\mathrm{s}} / \mu_{\mathrm{s}}$ & 0.0859 & 0.2378 & 0.2062 & 0.1708 & 0.5911 \\
\hline $\mathrm{n}_{\mathrm{s}}$ & 3.7781 & 4.0565 & 5.5807 & 4.6375 & 7.8500 \\
\hline$\beta_{\mathrm{s}}$ & 1.6359 & 1.5738 & 1.6349 & 1.5918 & 1.6929 \\
\hline$\mu_{b}(n m)$ & 3.9530 & 2.7297 & 2.4405 & 4.5179 & 2.7447 \\
\hline$b_{i}$ & & & & & 0.7379 \\
\hline$q_{b c i}$ & & & & & 0.2646 \\
\hline$\lambda_{\mathrm{bci}}$ & & & & & 0.7852 \\
\hline$a_{0}$ & 16.6217 & 21.799 & -0.7828 & 15.1430 & 171.687 \\
\hline$b_{0}$ & 0.8502 & 0.8914 & 0.1461 & 1.2497 & 4.5324 \\
\hline$q_{b c 0}$ & 0.1778 & 0.1773 & 0.1016 & 0.2444 & 0.4362 \\
\hline$\lambda_{\mathrm{bc} 0}$ & 3.5736 & 3.8401 & 1.1065 & 2.3170 & 1.6812 \\
\hline$\mu_{\mathrm{s}} / \mu_{\mathrm{b}}$ & 0.7843 & 1.1235 & 1.2846 & 0.7345 & 0.6139 \\
\hline$Z_{\text {Bethe }}$ & 6.62 & 6.64 & 10.84 & 5.09 & 3.29 \\
\hline
\end{tabular}

Sample identifiers

Sample\# A: PICA-20\%

(Without porogens)

Sample\# B: PICA-P1-1:1

$\mathrm{P} 1=$ Fumed silica

Sample\# C: PICA-P2-1:1

P2=ME48040M2

Sample\# D: PICA-P3-1:1

$\mathrm{P} 3=\mathrm{ME} 98040 \mathrm{M} 1$

Sample\# E: PICA-P4-2:1

P4=ME09730

PICA, polymer-induced colloid aggregation.

may be slit- or plate-type of a high-pore angularity. Figures 4 and 8 support this fact, as the isotherms abruptly increase at the higher partial pressure range. A moderately connected pore network and a small Bethe coordination number is found.

Sample C, PICA-P2-1:1: Figures 12a and b show convergence curves for PICA-P2-1:1. The psd and tsd (see Figures 5 or 8 and 9) are unimodal. Micropores are present; the material is in-homogeneous in construction; throat sizes are smaller than the pores, with narrow pore and throat size distributions; a moderately connected pore network with a low coordination number and a comparatively large Bethe coordination number is found.

Sample D, PICA-P3-1:1: Figures 13a and b show convergence curves for PICA-P3-1:1. The psd and tsd (see Figures 6 or 8 and 9) are unimodal. A large quantity of micropores are present in a rather homogeneous structure $\left(D_{\mathrm{s}}=2.0597\right)$. The aspect ratio of pores to throats is $>1$; the throat radius distribution is very narrow compared with that of the pores; the pore network is moderately connected, with low coordination numbers. 


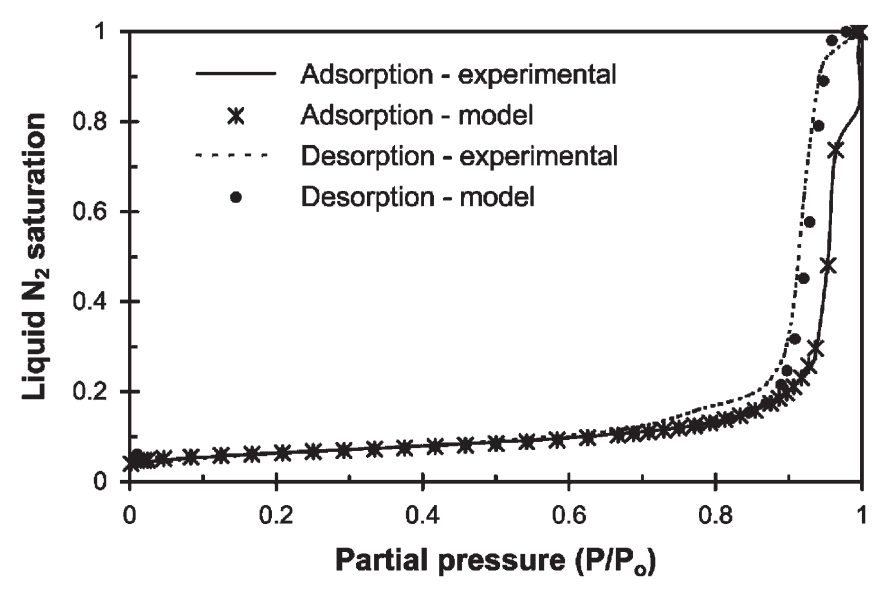

Figure 10. Experimental and model-predicted adsorption/desorption curves for Sample A, polymer-induced colloid aggregation particles made from $20 \%$ zirconia sol.

Sample E, PICA-P4-2:1: Figures 14a and b show convergence curves for PICA-P4-2:1. The psd and tsd (see Figures 7 or 8 and 9) are bimodal (secondary drainage
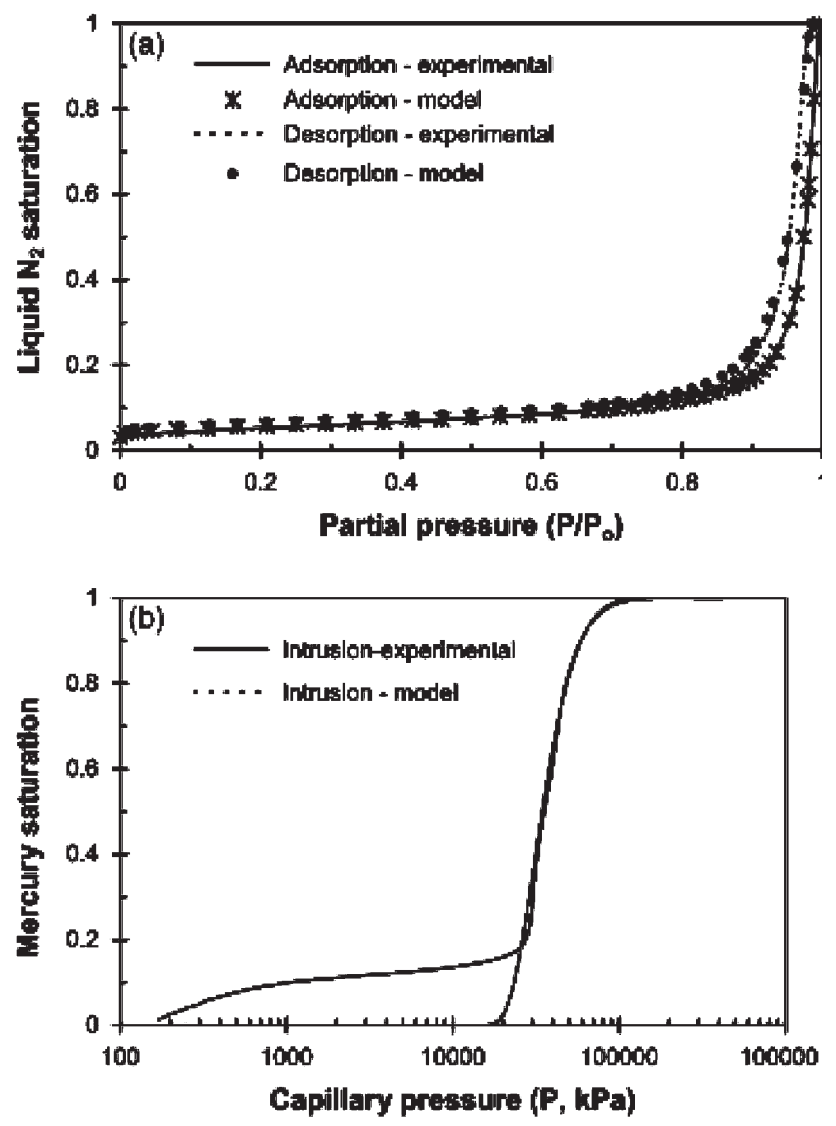

Figure 11. Experimental and model-predicted curves for Sample B, polymer-induced colloid aggregation (PICA) particles made with fumed silica in the ratio of 1:1 (PICA-P1-1:1). (a) Adsorption/desorption; (b) mercury intrusion. curves are present). However, for simplicity in calculations, the pores were modeled using a unimodal size distribution function. The sample is homogeneous in structure, having probably the largest quantity of micropores. The aspect ratio of pores to throats is less than one, with a narrow pore radius distribution and a very broad throat radius distribution, a moderately connected pore network, no strong $c$ - $t$-type correlation $\left(\lambda_{\mathrm{bc} 0}=1.6812, \lambda_{\mathrm{bci}}=0.7852\right)$, and a low Bethe coordination number $\left(Z_{\text {Bethe }}=3.2\right)$.

\section{Discrepancy between Actual and Model-Predicted Values}

The present method produces narrower pore- and throat-radius distributions with lower mean values compared with pore (or throat) size estimates available from the classical tube-bundle model. The reasons established for this discrepancy are as follows: (1) The volume of the pore or throat does not include
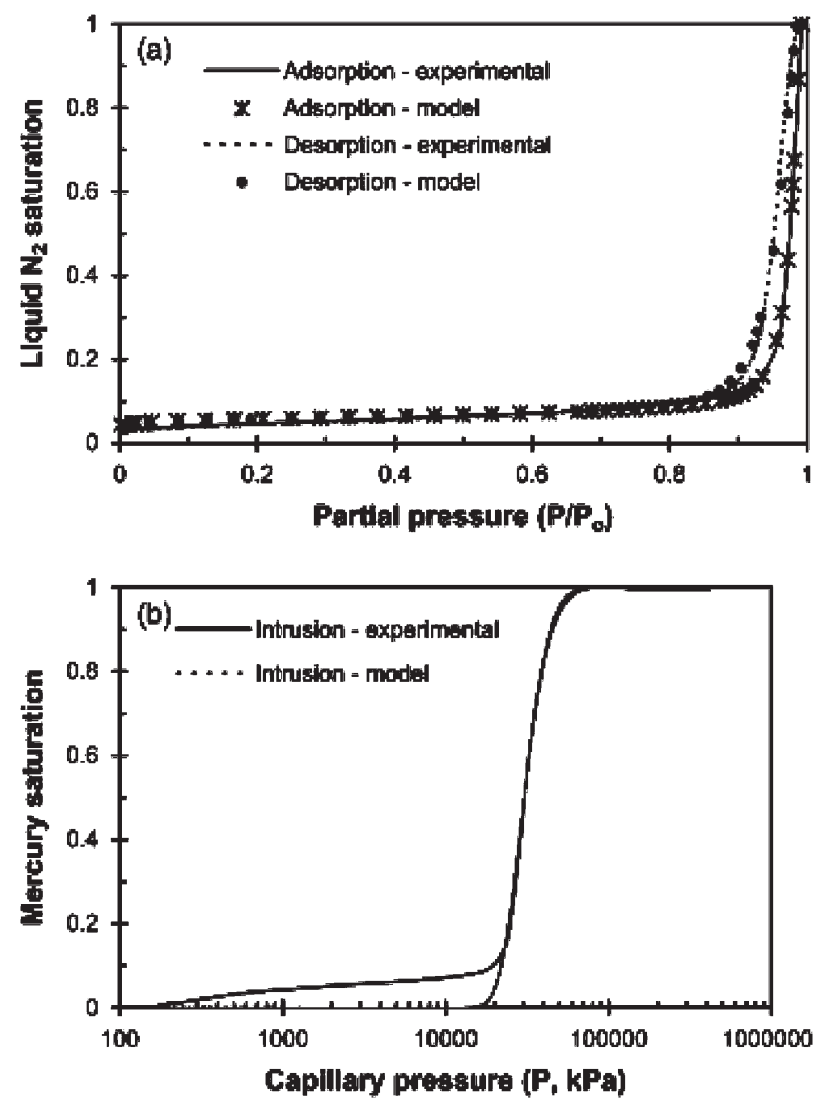

Figure 12. Experimental and model-predicted curves for Sample C, polymer-induced colloid aggregation (PICA) particles made with microcrystalline wax-ME48040M2 in the ratio of 1:1 (PICA-P2-1:1): (a) Adsorption/desorption; (b) mercury intrusion. 

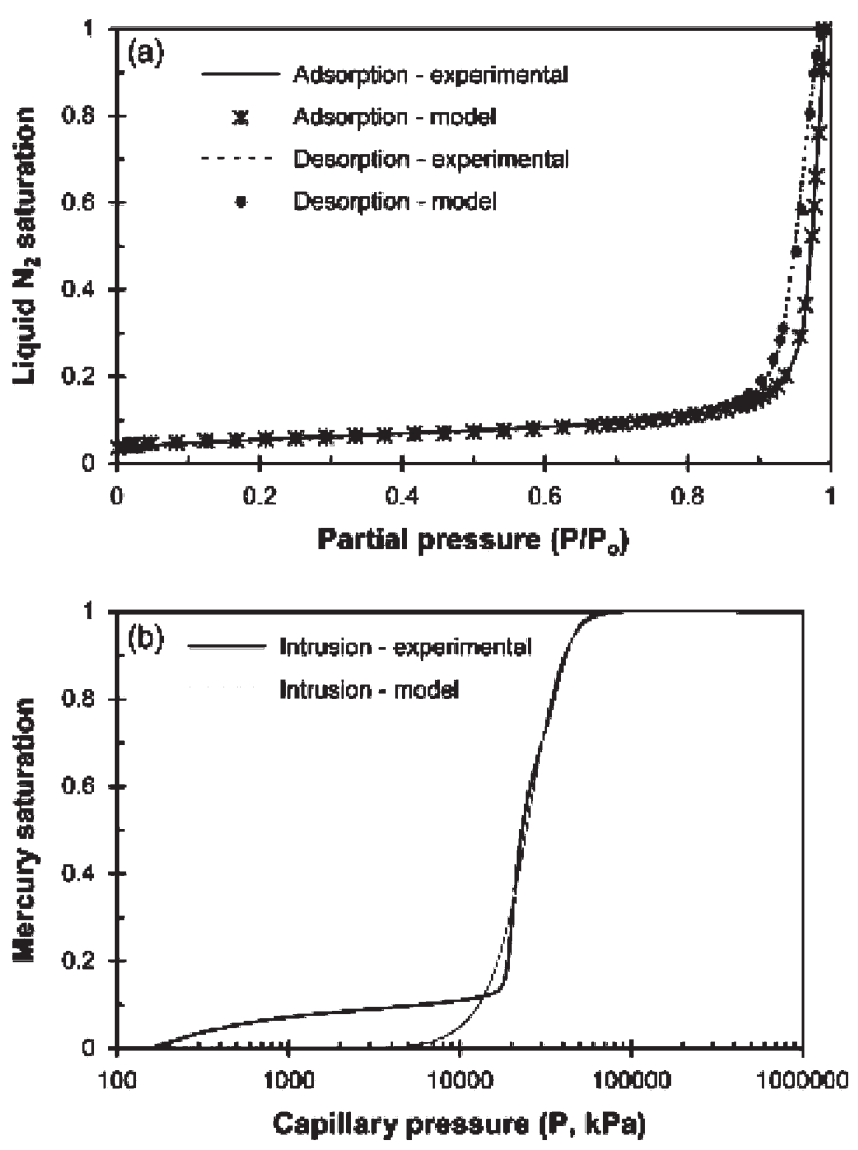

Figure 13. (a) Experimental and model-predicted curves for Sample D, polymer-induced colloid aggregation (PICA) particles made with microcrystalline wax-ME98040M1 in the ratio of 1:1 (PICA-P3-1:1): (a) adsorption/desorption; (b) mercury intrusion.

the volume contained in the pore cusps; thus a significant portion of pore or throat volume remains unaccounted for in the calculation of pore or throat sizes. To illustrate this situation better, a closer look at the pore or throat chamber construction might be helpful (Figures 15a and b). In Figure 15a, if the inside of the chamber can be described well by a regular polygon, then the effective chamber radius calculated by the present methodology is equal to the radius of the inscribed circle in the polygon. A major volume (represented by shaded area in the figure) remains disregarded in model calculations. However, as was true with most of the samples analyzed in this report, the pore-network system was predominantly constituted of slit- or plate-shaped channels $\beta_{\mathrm{s}}<2$ ) (Figures 15c-e for types of pores). The cross-section of such a channel might look similar to Figure 15b. The volume encompassed by such a system might be enormous, as determined by the classical tube-bundle model; how-
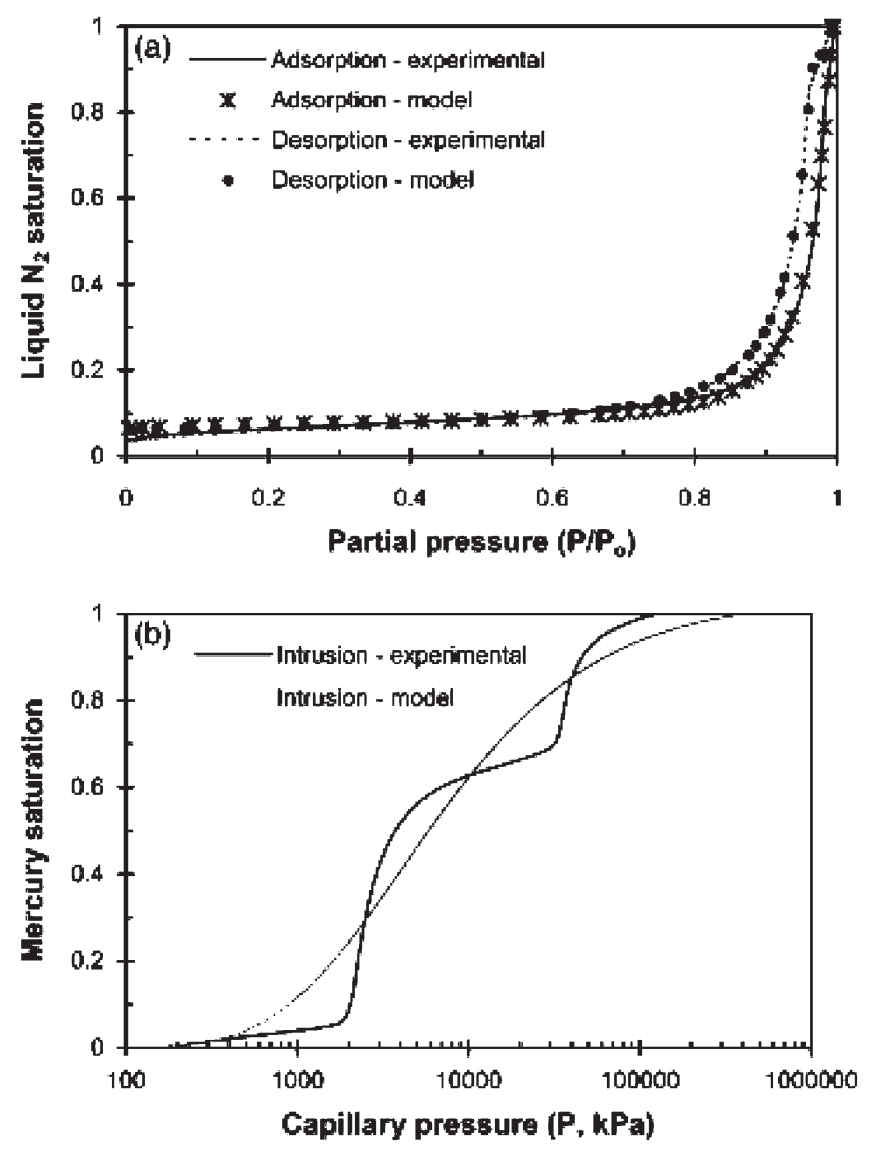

Figure 14. (a) Experimental and model-predicted curves for Sample E, polymer-induced colloid aggregation (PICA) particles made with microcrystalline wax-ME09730 in the ratio of 2:1 (PICA-P4-2:1): (a) adsorption/desorption; (b) mercury intrusion.

ever, the true radius calculated from the present model is significantly smaller (represented by the inscribed blank circle). This radius signifies the size of the largest particle of regular dimension that can pass through the channel unhindered. Interestingly, in Figure 15b, two such blank circles may be fitted side-by-side within the pore or throat cross-section. Nevertheless, the effective radius of the pore system will remain unaffected, signifying that the mass flux through the crosssection may be higher in Figure $15 \mathrm{~b}$ as compared with Figure 15a, and only objects smaller than this radius can pass through. (2) For samples that revealed sorption isotherms of Type-IV and intermediate of $\mathrm{H} 1$ and $\mathrm{H} 3$, the abrupt volume change toward the higher partial pressure gave rise to model incompatibility, and therefore, the simulation was not free from unambiguous results. Additionally, the adsorption branch often led to smaller pore values compared with the desorption branch. This defect was apparent for materials 

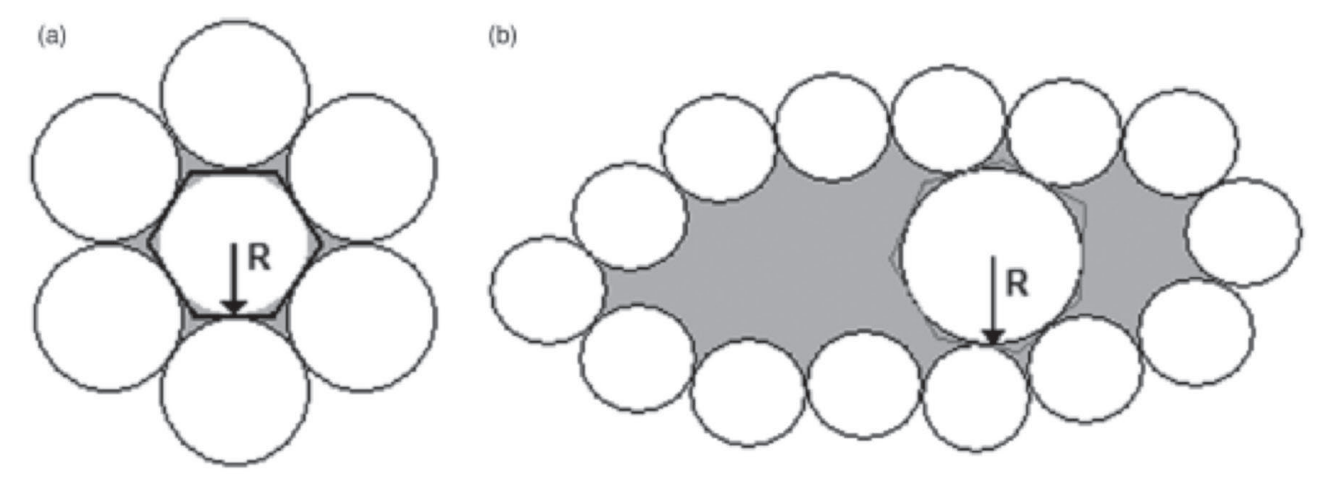

(c)
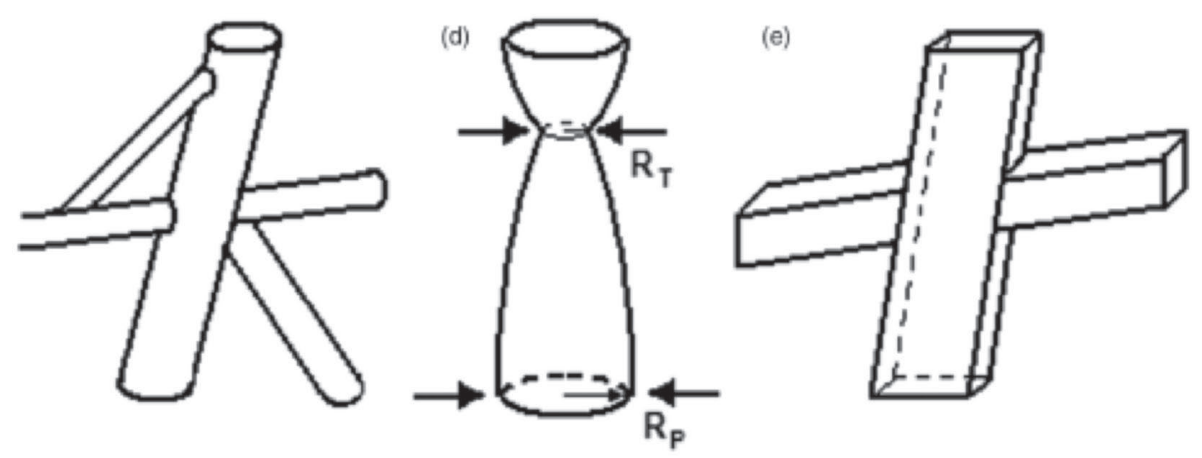

Figure 15. (a) Equivalent chamber radius in a cylindrical pore or throat; (b) Equivalent chamber radius in a slit- or plate-type channel; Types of pores: (c) cylindrical, (d) conical/ink-bottle-shaped, (e) slit- or plate-type.

having mesopores, as the nitrogen sorption method works well only for pore or throat diameters $<100 \mathrm{~nm}$. The PSDs for these samples revealed that the distributions were abruptly incomplete beyond the highest peak of the distribution curves (pore diameters beyond $100 \mathrm{~nm}$ ). (3) For the calculation of pore sizes, only the adsorption branch was de-convoluted. However, most of the samples had pore dimensions that were beyond the range of determination by sorption, and some samples had a bimodal distribution, too. The corresponding mercury extrusion curves showed the true picture of pore-size distribution. Thus, the estimated pore values were smaller than the probable true values. (4) The discrepancy observed over the low-pressure region of mercury intrusion, where the experimental curve did not merge with the model-predicted one, can be attributed to surface pores that were accessible through large throats. The entire pore structure may be heterogeneous, consisting of a uniform network of fine pores surrounded by a thin layer of large pores. Therefore, a bimodal pore or throat size distribution and a composite accessibility function might have helped in better estimating the parameters. ${ }^{37}$ However, because of the complex nature of the present model, where the number of unknown variables is large, the introduction of a bimodal function in the model would have involved additional variables, and parameter estimation would have become more complicated. To maintain the userfriendliness of this model, all samples were assumed to be unimodal. (5) The present model is very sensitive to the choice of initial values for the variables and also to the degree of accuracy of values desired. The SSR minimization produces more than one set of optimum parameter values; thus, the selection of initial values is crucial. Finally, inherent limitations of any simple geometric pore model restrict the precise determination of complex pore structures.

\section{Summary}

Our present study suggests that the optimum ratio of zirconia and porogen is largely decided by the hydrodynamic size of the porogen, the physical and chemical properties of the porogens, the self-associative properties of the porogens, the concentration of the sol, and the concentrations of urea and formaldehyde. Also, it is revealed that the shapes of pores for all the experiments in this study were near-cylindrical or slit types, debarring a few exceptions, in which either the zirconia sol content was too low or the porogen loading was too high. While porogens fumed sil- 
ica and MCW could only produce pores in the range of 100-200 nm, the polyethylene emulsion avoided this limitation and produced distinct, hierarchically sized pores in the ranges $10-110$ and $600-3000 \mathrm{~nm}$, owing to its higher melting point and irrespective of its small porogen size. Thus, depending on the pore size requirement, a suitable porogen may be chosen for synthesizing porous zirconia supports.

\section{Acknowledgements}

The technical assistance of the staff at the Center of Materials Research and the Microscopy facility at the University of Nebraska-Lincoln is greatly appreciated.

\section{References}

1. H. Chen and Cs. Horvath, "High-Speed High-Performance Liquid Chromatography of Peptides and Proteins," J. Chromatogr. A, 705 [1] 3-20 (1995).

2. M. J. Annen, R. Kizhappali, P. W. Carr, and A. V. McCormick, “Development of Porous Zirconia Spheres by Polymerization-Induced Colloid Aggregation," J. Mater. Sci., 29 [23] 6123-6130 (1994).

3. A. M. Clausen, A. Subramanian, and P. W. Carr, "Purification of Monoclonal Antibodies from Cell Culture Supernatants Using a Modified Zirconia Based Cation-Exchange Support," J. Chromatogr. A, 831 [1] 63-72 (1999).

4. A. Subramanian, P. W. Carr, and C. V. McNeff, "Use of SprayDried Zirconia Microspheres in the Separation of Immunoglobulins from Cell Culture Supernatant," J. Chromatogr. A, 890 [1] 15$23(2000)$

5. G. J. Soler-Illia, C. Sanchez, B. Lebeau, and J. Patarin, "Chemical Strategies to Design Textured Materials: From Microporous and Mesoporous Oxides to Nanonetworks and Hierarchical Structures," Chem. Rev., 102 [11] 4093-4138 (2002).

6. U. Trudinger, G. Muller, and K. K. Unger, "Porous Zirconia and Titania as Packing Materials for HPLC," J. Chromatogr., 535 [1] 111125 (1990).

7. R. A. Shalliker, G. K. Douglas, P. R. Comino, and P. E. Kavanagh, "Examination of Various Pore Size Zirconias for Potential Chromatographic Applications," Powder Technol., 91 [1] 17-23 (1997).

8. R. A. Shalliker and G. K. Douglas, "Controlling the Pore Structure of Zirconia for Chromatographic Applications," J. Liq. Chromatogr. Related Technol., 20 [11] 1651-1666 (1997).

9. C. Verissimo and O. L. Alves, "Microstructural Modifications in Macroporous Oxides Prepared Via Latex Templating: Synthesis and Thermal Stability of Porous Microstructure," J. Am. Ceram. Soc., 89 [7] 2226-2231 (2006).

10. J. H. Schattka, E. H.-M. Wong, M. Antonietti, and R. A. Caruso, "Sol-Gel Templating of Membranes to Form Thick, Porous Titania, Titania/Zirconia and Titania/Silica Films," J. Mater. Chem., 16 [15] 1414-1420 (2006).

11. J. H. Schattka, D. G. Shchukin, J. Jia, M. Antonietti, and R. A. Caruso, "Photocatalytic Activities of Porous Titania and Titania/ Zirconia Structures Formed by Using a Polymer Gel Templating Technique," Chem. Mater., 14 [12] 5103-5108 (2002).

12. D. G. Shchukin and R. A. Caruso, "Template Synthesis and Pho- tocatalytic Properties of Porous Metal Oxide Spheres Formed by Nanoparticle Infiltration," Chem. Mater., 16 [11] 2287-2292 (2004).

13. F. Tang, H. Fudouzi, T. Uchikoshi, and Y. Sakka, "Preparation of Porous Materials with Controlled Pore Size and Porosity," J. Eur. Ceram. Soc., 24 [2] 341-344 (2004).

14. Y. Xia and R. Mokaya, "Hollow Spheres of Crystalline Porous Metal Oxides: A Generalized Synthesis Route Via Nanocasting with Mesoporous Carbon Hollow Shells," J. Mater. Chem., 15 [30] 3126-3131 (2005).

15. V. Suryanarayanan, A. Sreekumaran, R. T. Tom, and T. Pradeep, "Porosity of Core-Shell Nanoparticles," J. Mater. Chem., 14 [17] 2661-2666 (2004).

16. X. X. Li, L. Chen, B. Li, and L. Li, "Preparation of Zirconia Nanopowders in Ultrasonic Field by the Sol-Gel Method," Key Eng. Mater., 280-283 981-986 (2005).

17. M. Wolff, G. Falk, R. Clasen, and G. T. Link, “Densification Behavior of Zirconia Ceramics Sintered Using High-Frequency Microwaves," Ceram. Eng. Sci. Proc., 27 [8] 373-380 (2005).

18. A. Imhof and D. J. Pine, "Uniform Macroporous Ceramics and Plastics by Emulsion Templating," Adv. Mater., 10 [9] 697-700 (1998).

19. R. Linacero, J. Aguado-Serrano, and M. L. Rojas-cervantes, "Preparation of Mesoporous $\mathrm{TiO}_{2}$ by the Sol-Gel Method Assisted by Surfactants," J. Mater. Sci., 41 [8] 2457-2464 (2006).

20. Y. K. Hwang, K. R. Patil, S. H. Jhung, J. Chang, Y. J. Ko, and S. Park, "Control of Pore Size and Condensation Rate of Cubic Mesoporous Silica Thin Films Using a Swelling Agent," Microporous Mesoporous Mater., 78 [2-3] 245-253 (2005).

21. T. Ren, Z. Yuan, and B. Su, "Hierarchical Microtubular Nanoporous Zirconia with an Extremely High Surface Area and Pore Volume," Chem. Phys. Lett., 388 [1-3] 46-49 (2004).

22. C. Y. Tai, M. Lee, and Y. Wu, "Control of Zirconia Particle Size by Using Two-Emulsion Precipitation Technique," Chem. Eng. Sci., 56 [7] 2389-2398 (2001).

23. J. He, T. Ma, Y. Huang, and J. Yang, "Study on Preparation of Spherical Zirconia Powder in Microemulsion System and Its Surface Characteristics," Key Eng. Mater., 280-283 977-980 (2005).

24. P. W. Carr, A. V. Mccormick, M. J. Annen, L. Sun, and J. R. Brown "Synthesis of Porous Inorganic Particles by Polymerization-Induced Colloid Aggregation (PICA)," U.S. Patent 5540834, 1996, 42pp.

25. L. Sun, M. J. Annen, F. Lorenzano-Porras, P. W. Carr, and V. McCormick, "Synthesis of Porous Zirconia Spheres for HPLC by Polymer-Induced Colloid Aggregation (PICA)," J. Colloid Interface Sci., 163 [1] 464-473 (1994).

26. M. S. J. Gani, et al. "Porous Zirconia Particles, and Manufacture of Porous Tetragonal Zirconia," PCT Int. Appl., 1-68 (1995).

27. S. Sarkar "Characterization and Optimization of EDTPA Zirconia based Chromatographic Process," M.S. Thesis, University of Minnesota, 2001.

28. A. Subramanian and S. Sarkar, "The Use of EDTPA-Modified Zirconia as a Preparative HPLC Support," J. Chromatogr., 944 [1-2] 179-187 (2002).

29. A. Subramanian and S. Sarkar, "Interaction of EDTPA-Modified Zirconia with Immunoglobulins," J. Chromatogr., 989 [1] 131-138 (2003).

30. S. Sarkar, C. V. McNeff, P. W. Carr, and A. Subramanian, "Characterization and Optimization of a Chromatographic Process Based on EDTPA-Modified Zirconia," J. Chromatogr., 790 [1-2] 143-152 (2003).

31. M. Leonard, "New Packing Materials for Protein Chromatography," J. Chromatogr. B, 699 [1-2] 3-27 (1997).

32. X. Wu, K. Haupt, and M. Vkjayalakshmi, "Separation of Immunoglobulin G by High-Performance Pseudo-Bioaffinity Chromatography with Immobilized Histidine," J. Chromatogr., 584 [1] 35-41 (1992). 
33. S. Sarkar and A. Subramanian, "Modeling of Immunoglobulin Uptake by $\mathrm{N}, \mathrm{N}, \mathrm{N}^{\prime}, \mathrm{N}^{\prime}$-Ethylenediaminetetramethylenephosphonic Acid-Modified Zirconia Particles Under Static and Dynamic Conditions," J. Chromatogr. B Analyt. Technol. Biomed. Life Sci., 821 [1] 81-87 (2005).

34. S. Sarkar, P. W. Carr, and A. Subramanian, "Identification of the Mass Transfer Mechanisms Involved in the Transport of Human Immunoglobulin-G in $\mathrm{N}, \mathrm{N}, \mathrm{N}^{\prime}, \mathrm{N}^{\prime}$-Ethylenediaminetetramethylenephosphonic Acid-Modified Zirconia," J. Chromatogr. B Analyt. Technol. Biomed. Life Sci., 821 [2] 124-131 (2005).

35. A. Mullick, C. M. Griffith, and M. C. Flickinger, "Expanded and Packed Bed Albumin Adsorption on Fluoride Modified Zirconia," J. Biotechnol. Bioeng., 60 [3] 333-40 (1998).

36. X. Wang, L. Liu, and A. J. Jacobson, "Open-Framework and Microporous Vanadium Silicates," J. Am. Chem. Soc., 124 [26] 78127820 (2002).

37. C. D. Tsakiroglou and A. C. Payatakes, "New Mercury IntrusionRetraction Simulator Used as a Means for the Characterization of Porous Materials," Stud. Surf. Sci. Catal., 62 [Charact. Porous Solids 2] 169-178 (1991).

38. C. D. Tsakiroglou, V. N. Burganos, and J. Jacobsen, "Pore Structure Analysis by Using Nitrogen Sorption and Mercury Intrusion Data," AI. Ch. E. J., 50 [2] 489-510 (2004).

39. C. D. Tsakiroglou and A. C. Payatakes, "Effects of Pore-Size Correlations on Mercury Porosimetry Curves," J. Colloid Interface Sci., 146 [2] 479-494 (1991).

40. B. Sahouli, S. Blacher, and F. Brouers, "Fractal Surface Analysis by Using Nitrogen Adsorption Data: The Case of the Capillary Condensation Regime," Langmuir, 12 [11] 2872-2874 (1996).

41. A. L. Ahmad, N. F. Idrus, and S. R. A. Shukor, "Surface Fractal Dimension of Perovskite-Doped Alumina Membrane: Influence of Calcining Temperature," J. Am. Ceram. Soc., 89 [5] 1694-1698 (2006).

42. P. Aparicio, J. L. Perez-Bernal, E. Galan, and M. A. Bello, "Kaolin Fractal Dimension. Comparison with Other Properties," Clay Miner., 39 [1] 75-84 (2004).

43. A. V. Neimark, M. Hanson, and K. K. Unger, "Fractal Analysis of the Distribution of High-Viscosity Fluids in Porous Supports," J. Phys. Chem., 97 [22] 6011-6015 (1993).

44. Y. Li, G. Q. Lu, and V. Rudolph, "Compressibility and Fractal Dimension of Fine Coal Particles in Relation to Pore Structure Characterization using Mercury Porosimetry," Part. Part. Syst. Char., 16 [1] 25-31 (1999).

45. B. Sahouli, S. Blacher, and F. Brouers, "Applicability of the Fractal FHH Equation," Langmuir, 13 [16] 4391-4394 (1997).

46. F. Ehrburger-Dolle, M. Holz, and J. Lahaye, "Use of Nitrogen, Argon and Carbon Dioxide Adsorption for the Determination of Microporosity and Surface Fractal Dimension of Carbon Blacks and Silicas," Pure Appl. Chem., 65 [10] 2223-2230 (1993).

47. J. Zhang and G. Zografi, “The Relationship Between BET- and Free Volume-Derived Parameters for Water Vapor Absorption into Amorphous Solids," J. Pharm. Sci., 89 [8] 1063-1072 (2000).

48. M. Sahimi, Applications of Percolation Theory, Taylor \& Francis, London, 1994.

\section{Appendix A}

\section{Pore Network Modeling-Mathematical Preliminaries}

A modeling exercise was performed on some select samples based on the application of a recently formulated pore-network modeling methodology ${ }^{37-39}$ to produce only one set of geometrical and topological parameters. This model suitably applies to porous systems comprised of sintered materials, palletized materials, and agglomerates that exhibit cusps rather than microroughness along pore walls. The original work was done by Tsakiroglou et al. ${ }^{38}$ in an ATHENA visual workbench software environment, which involved a rigorous iterative simulation on experimental sorption and porosimetry data in order to match experimental curves with analytical curves derived from the model. This report performs a similar modeling in a MATLAB 6 environment. In addition, the present study also takes into account the effect of surface fractal dimension on the shape determination of sorption isotherms ( $s$, a key shape-determining factor in the Frenkel-Halsley-Hill (FHH) equation), something that was considered constant $(s=2)$ in the previous work. The model was evaluated assuming uncorrelated and $c$ - $t$-correlated networks only. The foregoing sections briefly discuss the various parameters involved in the model and their physical significance.

Surface Fractal Dimension $\left(D_{s}\right)$ : The fractal dimension describes how an object fills its space, ${ }^{40}$ or measures the surface roughness, ${ }^{41,42}$ and heterogeneity or irregularity. ${ }^{42}$ According to Eucledian geometry, $D_{\mathrm{s}}=0$ represents a point; $D_{\mathrm{s}}=1$ represents a curve; $D_{\mathrm{s}}=2$ represents a surface; and $D_{\mathrm{s}}=3$ represents a volume. A higher $D_{\mathrm{s}}$ value corresponds to a rougher surface. Generally, fractal dimension values lie between 2 and 3 . Usually, $D_{\mathrm{s}}$ is determined from the adsorption data by FHH, the Pfeifer-Avnir or the thermodynamic method proposed by Neimark, ${ }^{43}$ or from mercury intrusion data. ${ }^{44}$ This report uses the Neimark's thermodynamic method. The average fractal dimension in the pore size range of 2-60 $\mathrm{nm}$ (region of linearity) was found for all the samples. The main equation of this method is

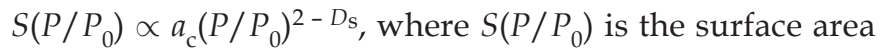
for a given value of relative pressure $\left(P / P_{0}\right), P_{0}$ the nitrogen saturation pressure, and $a_{\mathrm{c}}$ the mean curvature radius at $\mathrm{P} / \mathrm{P}_{0}$. The surface area of the film is calculated using the Kiselev integral equation, ${ }^{40} S\left(P / P_{0}\right)=(R T / \gamma) \int_{V\left(P / P_{0}\right)}^{N_{\max }}$ $\ln \left(P / P_{0}\right) \mathrm{d} N$ where $N_{\max }$ denotes the amount of adsorbate when $P / P_{0}$ tends toward unity and $\gamma$ is the surface tension of the liquid adsorbate (nitrogen), $R$ the gas constant, and $T$ the temperature (in Kelvin). The Kelvin equation is used to calculate the mean radius at a partial pressure $a_{\mathrm{c}}\left(P / P_{0}\right)$ $=\left(2 \gamma V_{\mathrm{m}}\right) /\left\{R T \ln \left(P / P_{0}\right)\right\}$, where $V_{\mathrm{m}}$ is the molecular volume of the adsorbate. The plot of $\log S\left(P / P_{0}\right)$ vs. $\log a_{c}(P /$ $\left.P_{0}\right)$ is a straight line in the fractal region with a $2-D_{\mathrm{s}}$ slope value, from which the $D_{\mathrm{s}}$ was finally calculated by minimizing the sum square error of the surface area. 
The FHH exponent, s: The FHH equation is considered as a shape-characterizing parameter for sorption isotherms in the multilayer region, similar to the $C_{\mathrm{BET}}$ constant in the monolayer region. For flat surfaces, the FHH equation reads as $\theta \propto\left[\ln \left(P_{0} / P\right)\right]^{-1 / s}$, where $\theta$ is the adsorbed quantity. The generalized version of the fractal $\mathrm{FHH}$ equation proposed by Pfeifer is $\theta \propto\left[\ln \left(P_{0} / P\right)\right]^{-1 / m}$, where $m=\mathrm{s} /(3$ $-D_{\mathrm{s}}$ ). When $s=3$, this equation is called the fractal FHH equation and it is valid only at the early stages of the multilayer formation, where nonretarded van der Walls interactions hold valid. The literature ${ }^{45-47}$ cites exhaustive discussions on the regimes of applicability of the generalized FHH equation, case studies for different values of $m$, the dependence of $\mathrm{m}$ on surface fractal property, and the microporosity and the chemical nature of the adsorbent. As a fact, mesoporosity decreases the FHH exponent, and microporosity increases it. For adsorption dominated by capillary condensation in materials devoid of micropores, $m$ is calculated as $m=1 /\left(3-D_{s}\right)$. When solid-gas potential controls adsorption, $\mathrm{m}$ is derived as $m=s /\left(3-D_{\mathrm{s}}\right)$. The high $C_{\mathrm{BET}}$ for all the samples necessitated the consideration of the effect of the presence of micropores. $C_{\mathrm{BET}}$ is a constant reflecting the overall free energy of adsorption and the chemical affinity of the adsorbate for the adsorbent. ${ }^{47}$ For the sake of simplicity and uniformity in the analysis of all the samples, the $s$ was assumed to be equal to 3 . The original FHH parameter $s$ in the determination of the thickness of the adsorbed layer, ${ }^{38} t_{c}=\sigma[\{b / b / \ln (1 / x)\}]^{(1 / s)}$, was replaced with $m$, where $m=(s=3) /\left(3-D_{s}\right)$.

The Pore (Chamber or Site) and Throat (Bond) Size Distributions: The very first assumption of this model is that the pores and throats are distributed randomly (uncorrelated networks) or nonrandomly (correlated networks) as a probabilistic function of the lognormal distribution of the forms for sites and bonds, respectively, as

and

$$
f_{\mathrm{s}}\left(r ; \mu_{\mathrm{s}}, \sigma_{\mathrm{s}}\right)=\frac{1}{r \sigma_{\mathrm{s}} \sqrt{2 \pi}} \exp \left[-\frac{\left\{\ln (r)-\mu_{\mathrm{s}}\right\}^{2}}{2 \sigma_{\mathrm{s}}^{2}}\right]
$$

$$
f_{\mathrm{b}}\left(r ; \mu_{\mathrm{b}}, \sigma_{\mathrm{b}}\right)=\frac{1}{r \sigma_{\mathrm{b}} \sqrt{2 \pi}} \exp \left[-\frac{\left\{\ln (r)-\mu_{\mathrm{b}}\right\}^{2}}{2 \sigma_{\mathrm{b}}^{2}}\right]
$$

where $r$ is the critical chamber radius at a given saturation pressure.

$\mu_{\mathrm{s}^{\prime}} \mu_{\mathrm{b}}$ is the mean value of the pore/throat size distribution. It indicates where the peak of the density occurs; $\sigma_{s^{\prime}} \sigma_{b}$ is the standard deviation of the pore/throat size distribution. It indicates the girth or spread of the probability distribution curve; $\mu_{\mathrm{s} /} \mu_{\mathrm{b}}$ is the mean aspect ratio of the pore-to-throat radius. An aspect ratio of 1 may mean cylindrical or parallelepiped pores; $\left(\sigma_{s} / \mu_{s}\right),\left(\sigma_{b} / \mu_{b}\right)$ measures the narrowness or broadness of the pore (or throat) radius distribution.

Pore and Throat Volumes: The throats are narrow constrictions without any volume, and the entire chamber volume is estimated by $V_{\mathrm{s}}(r) \propto r^{\beta_{\mathrm{s}}}$, where $\beta_{\mathrm{s}}$ is the pore volume exponent or the volume shape factor. Necessarily, $\beta_{\mathrm{s}}$ $\geq 0 . \beta_{\mathrm{s}}=2.0$ represents long capillaries of identical shape, whereas $\beta_{\mathrm{s}}=3.0$ indicates pores formed between interconnecting spherical particles. Importantly, for all the samples analyzed in this report, $\beta_{\mathrm{s}}$ values were in the range 1.551.70 , which may be indicative of slit- or plate-type pores. This conclusion is corroborated by the nature of the nitrogen adsorption/desorption curves, which were Type-IV but intermediate-type $\mathrm{H} 1$ and $\mathrm{H} 3$. As a first hand guess, $\beta_{\mathrm{s}}$ was set to 2 for the model.

Pore wall Geometry: Theoretically, the pore walls may be modeled with an imaginary high-order regular polygon having $n_{\mathrm{s}}$ sides, where each side may be considered a tangent to the constituting adjacent particles. It is regarded as a measure of the pore wall angularity (or fraction of porosity belonging to pore edges) rather than as an accurate geometrical representation of the pore shape. From a geometrical point of view, three sides are necessary to construct a polygon, so $n_{\mathrm{s}} \geq 3$ is a physical constraint. A value of $n_{\mathrm{s}}$ close to 3 indicates a high angular porosity. At an infinite value of $n_{\mathrm{s}^{\prime}}$ the pore wall crosssection is circular.

The $\mathrm{N}_{2}$ Adsorption/Desorption Models: The Kelvin equation estimates the curvature radius of the vapor/liquid menisci of the condensate, $r_{\mathrm{c}^{\prime}} r_{\mathrm{c}}=\left(2 V_{L}^{0} \gamma_{L G}\right) /\{R T \ln (1 / x)\}$. Finally, the liquid nitrogen saturation in the pore network as a function of relative vapor pressure is expressed in the integral form as

$$
\begin{aligned}
& S_{\mathrm{LN} 2}(r)= \\
& \frac{\int_{0}^{r_{\mathrm{s}}} f_{\mathrm{s}}(r) V_{\mathrm{s}}(r) \mathrm{d} r+\frac{q_{\mathrm{s}}-Y_{\mathrm{s}}\left(q_{\mathrm{b}}\right)}{q_{\mathrm{s}}-q_{\mathrm{si}}} \int_{r_{\mathrm{s}}}^{r_{\mathrm{s}}} f_{\mathrm{s}}(r) V_{\mathrm{s}}(r) \mathrm{d} r+\frac{Y_{\mathrm{s}}\left(q_{\mathrm{b}}\right)}{q_{\mathrm{s}}} \int_{r_{\mathrm{s}}}^{\infty} f_{\mathrm{s}}(r) V_{\mathrm{s}}(r) S_{f 2}(r) \mathrm{d} r}{\int_{0}^{\infty} f_{\mathrm{s}}(r) V_{\mathrm{s}}(r) \mathrm{d} r}
\end{aligned}
$$

The Mercury Intrusion Model: Mercury imbibition into pores is analogous to drainage from capillaries due to evaporation, and is controlled by throat sizes. This process is similar to primary desorption, such that $q_{s i}=0$. The critical radius of curvature of mercury intrusion at a given capillary pressure $\left(P_{\mathrm{c}}\right)$ is $r_{\mathrm{c}}=\gamma_{\mathrm{Hg}} / P_{\mathrm{c}}$. The mercury saturation in pore networks as a function of capillary pressures is expressed in the integral form as

$$
\begin{aligned}
& S_{\mathrm{Hg}}\left(P_{\mathrm{c}}\right)=1- \\
& \frac{\int_{0}^{r_{\mathrm{s}}} f_{\mathrm{s}}(r) V_{\mathrm{s}}(r) \mathrm{d} r+\frac{q_{\mathrm{s}}-Y_{\mathrm{s}}\left(q_{\mathrm{b}}\right)}{q_{\mathrm{s}}} \int_{r_{\mathrm{s}}}^{\infty} f_{\mathrm{s}}(r) V_{\mathrm{s}}(r) \mathrm{d} r+\frac{Y_{\mathrm{s} 0}\left(q_{\mathrm{b}}\right)}{q_{\mathrm{s}}} \int_{r_{\mathrm{s}}}^{\infty} f_{\mathrm{s}}(r) V_{\mathrm{s}}(r) S_{f 1}(r) \mathrm{d} r}{\int_{0}^{\infty} f_{\mathrm{s}}(r) V_{\mathrm{s}}(r) \mathrm{d} r}
\end{aligned}
$$


The Accessibility Function: The accessibility function below, which represents a sigmoid curve, includes porespace topology and spatial pore-size correlation information for the primary drainage $Y_{s 0}$ and secondary desorption $Y_{s i} \cdot$

$$
\begin{aligned}
Y_{\mathrm{s} i}\left(q_{\mathrm{b}}\right)= & \left\{q_{\mathrm{s} i}^{0}+\left(1+a_{i}-q_{\mathrm{s} i}^{0}\right) e^{-b_{i}\left(1-q_{\mathrm{b}}\right) / q_{\mathrm{b}}}\right\} / \\
& \left\{1+a_{i} e^{-b_{i}\left(1-q_{\mathrm{b}}\right) / q_{\mathrm{b}}}\right\}
\end{aligned}
$$

where $q_{s i}^{0}=0$ is the initial fraction of sites occupied by the nonwetting phase. For the model, it has been assumed that $q_{s i}^{0}=0$ for primary drainage and desorption, and $q_{s i}^{0}=q_{s i}$ for secondary desorption, initiating before the completion of adsorption.

$a_{i}, a_{0}$ is the proexponential factor involved in the accessibility function. Subscript " 0 " denotes the case with primary drainage or imbibition, and " $i$ " for secondary; $b_{i}, b_{0}$ is the exponent involved in secondary/primary accessibility function.

The accessibility function also gives quantitative measures of two parameters: (1) the percolation threshold, $q_{\mathrm{b}}=$ $q_{\mathrm{bci}}$, which corresponds to the inflection point of the curve $\left(d^{2} Y_{s i} / d q_{\mathrm{b}}^{2}\right)_{q_{\mathrm{b}}=q_{\mathrm{bci}}}=0$; and (2) the slope of the accessibility function at the percolation threshold, $\lambda_{\mathrm{bci}}$. These two parameters are obtained from the simultaneous numerical solution of the following three relations:

$$
\begin{aligned}
& b_{i}-2 z_{\mathrm{c}}-\left(2 z_{\mathrm{c}}+b_{i}\right) a_{i} e^{-b_{i} / z_{\mathrm{c}}}=0, \\
& \lambda_{\mathrm{bc} i}=\frac{b_{i}\left(a_{i}+1\right)\left(1-q_{\mathrm{s} i}\right) e^{-b_{i} / z_{\mathrm{c}}}}{z_{\mathrm{c}}^{2}\left(1+a_{i} e^{-b_{i} / z_{\mathrm{c}}}\right)^{2}}, \\
& z_{\mathrm{c}}=\frac{q_{\mathrm{bc} i}}{1-q_{\mathrm{bc} i}}
\end{aligned}
$$

$q_{\mathrm{bc} 0}, q_{\mathrm{bci}}$ is the bond percolation threshold in secondary or primary drainage. It is the smallest value at which spanning paths suddenly emerge and transform a random disconnected network to a connected one. Values of $q_{\mathrm{bc} 0}$ (or $q_{\mathrm{bci}}$ ) close to 0 may be interpreted as indicative of well-connected pores. Additionally, the $q_{\mathrm{bc} 0}\left(\right.$ or $q_{\mathrm{bc} i}$ ) value is further used to estimate the coordination number $\left(Z_{\text {Bethe }}\right)$ in a Bethe lattice ${ }^{48}$ as $q_{\mathrm{bc} 0}=1$ / $\left(Z_{\text {Bethe }}-1\right)$.

$\lambda_{\mathrm{bc} 0^{\prime}} \lambda_{\mathrm{bc} i}$ is the slope of the primary/secondary accessibility function at the percolation threshold. A high value indicates a high mean coordination number of throats to pores.

Interconnectedness of $a_{0}, b_{0}, q_{b c 0}, \lambda_{b c 0}$ : For a system of parallel pores of infinite connectivity, $q_{\mathrm{bc} 0}$ tends to 0 and $\lambda_{\mathrm{bc} 0}$ tends to infinity (or $a_{0}$ tends to -1 and $b_{0}$ tends to 0$).{ }^{37}$ A similar interpretation also holds for the secondary desorption parameters of $a_{\mathrm{i}^{\prime}}, b_{\mathrm{i}^{\prime}} q_{\mathrm{bci}}$ and $\lambda_{\mathrm{bc} i}$. 